Morphisms in the Category of Finite Dimensional Absolute Valued Algebras

Seidon Alsaody 



\title{
MORPHISMS IN THE CATEGORY OF FINITE DIMENSIONAL ABSOLUTE VALUED ALGEBRAS
}

\author{
SEIDON ALSAODY
}

\begin{abstract}
This is a study of morphisms in the category of finite dimensional absolute valued algebras, whose codomains have dimension four. We begin by citing and transferring a classification of an equivalent category. Thereafter, we give a complete description of morphisms from one-dimensional algebras, partly via solutions of real polynomials, and a complete, explicit description of morphisms from two-dimensional algebras. We then give an account of the reducibility of the morphisms, and for the morphisms from two-dimensional algebras we describe the orbits under the actions of the automorphism groups involved. Parts of these descriptions rely on a suitable choice of a cross-section of four-dimensional absolute valued algebras, and we thus end by providing an explicit means of transferring these results to algebras outside this crosssection.
\end{abstract}

\section{Definitions And Background}

An algebra $A=(A, \cdot)$ over a field $k$ is a vector space $A$ over $k$ equipped with a $k$-bilinear multiplication $A \times A \rightarrow A,(x, y) \mapsto x y=x \cdot y$. Neither associativity nor commutativity is in general assumed. $A$ is called unital if it contains an element neutral under multiplication; in that case, such an element is unique, and will be denoted by 1 . If $A$ is non-zero, and if for each $a \in A \backslash\{0\}$, the maps $L_{a}: A \rightarrow$ $A, x \mapsto a x$ and $R_{a}: A \rightarrow A, x \mapsto x a$ are bijective, $A$ is called a division algebra. This implies that $A$ has no zero divisors and, if the dimension of $A$ is finite, it is equivalent to having no zero divisors.

An algebra $A$ is called absolute valued if the vector space is real and equipped with a norm $\|\cdot\|$ such that $\|x y\|=\|x\|\|y\|$ for all $x, y \in A$. By [1] the norm in a finite dimensional absolute valued algebra is uniquely determined by the algebra multiplication if the algebra has finite dimension. The multiplicativity of the norm imlplies that an absolute valued algebra has no zero divisors and hence, if it is finite dimensional, that it is a division algebra. The class of all finite dimensional absolute valued algebras forms a category $\mathcal{A}$, in which the morphisms are the nonzero algebra homomorphisms. Thus $\mathcal{A}$ is a full subcategory of the category $\mathcal{D}(\mathbb{R})$ of finite dimensional real division algebras. It is known that morphisms in $\mathcal{A}$ respect the norm, and are hence injective. (Injectivity in fact holds for all morphisms in $\mathcal{D}(\mathbb{R})$.)

2010 Mathematics Subject Classification. 17A35; 17A80.

Key words and phrases. Absolute valued algebra, division algebra, homomorphism, irreducibility, composition.

Note: This is a preprint of "S. Alsaody, Colloq. Math. 125 (2011), 147-174". The copyright of this work is held by the Institute of Mathematics, Polish Academy of Sciences, and this version is available here with the publisher's permission. 


\subsection{Notation.}

1.1.1. Complex Numbers and Quaternions. The real and imaginary part of $A \in$ $\{\mathbb{C}, \mathbb{H}\}$ will be denoted by $\Re A$ and $\Im A$, respectively. We also use the notation $a=\Re(a)+\Im(a)$ for elements $a \in A$. The letters $\mathbf{i}, \mathbf{j}, \mathbf{k}$ denote the standard basis of the imaginary space $\Im \mathbb{H}$ of the quaternion algebra $\mathbb{H}$, and $\mathbf{i}$ will also be used as the imaginary unit in $\mathbb{C}$ as confusion is improbable. Complex and quaternion conjugation (negation of the imaginary part) will be denoted by $x \mapsto \bar{x}$ for $x \in \mathbb{C}$ or $x \in \mathbb{H}$. A quaternion with vanishing imaginary part and real part $r$ is simply denoted by $r$ in view of the embedding of $\mathbb{R}$ into $\mathbb{H}$, and the notation $\mathbb{S}(\mathbb{H})$ and $\mathbb{S}(\Im \mathbb{H})$ will be used for the set of quaternions of norm one and the set of purely imaginary quaternions of norm one, respectively. For $p \in \mathbb{S}(\mathbb{H})$ we have $p^{-1}=\bar{p}$, and we will denote the map $x \mapsto p x p^{-1}=p x \bar{p}$ by $\kappa_{p}$ and refer to it as conjugation by $p$.

1.1.2. Other Conventions. Throughout the paper, the abbreviations $\nu_{c}:=\cos \nu$ and $\nu_{s}:=\sin \nu$ will be used to enhance readability, as trigonometric expressions are abundant in many equations, where at the same time the trigonometry itself is of little importance.

Moreover, the elements 1 and -1 of the cyclic group $C_{2}$ will often be written simply as + and - , respectively. If $n$ is a positive integer, the notation $\underline{n}=\{k \in$ $\mathbb{N} \mid 1 \leq k \leq n\}$ will be used. Square brackets [ ] around a sequence of vectors will denote their span, whereas $\langle$,$\rangle denotes the following inner product of two$ quaternions: given $x=s_{0}+s_{1} \mathbf{i}+s_{2} \mathbf{j}+s_{3} \mathbf{k}$ and $x^{\prime}=s_{0}^{\prime}+s_{1}^{\prime} \mathbf{i}+s_{2}^{\prime} \mathbf{j}+s_{3}^{\prime} \mathbf{k}$, set $\left\langle x, x^{\prime}\right\rangle=\sum_{i=0}^{3} s_{i} s_{i}^{\prime}$. The norm of the absolute valued algebra $\mathbb{H}$ is then given by $\|x\|=\sqrt{\langle x, x\rangle}$ for all $x \in \mathbb{H}$.

Finally, given a category $\mathcal{C}$, and objects $A, B \in \mathcal{C}$, the class of morphisms in $\mathcal{C}$ from $A$ to $B$ is denoted $\mathcal{C}(A, B)$. Given a group $G$ acting from the left on a set $S$, we denote by ${ }_{G} S$ the category whose object class is $S$, and in which for $x, y \in S$, a morphism from $x$ to $y$ is a triple $(x, y, g)$ such that $g \cdot x=y$. When the objects $x$ and $y$ are clear from context, we will denote such a morphism simply by $g$ to avoid cumbersome notation.

1.2. History and Outline. In 1947, Albert characterized all finite dimensional absolute valued algebras as follows. [1]

Proposition 1.1. Every absolute valued algebra is isomorphic to an orthogonal isotope $(A, \cdot)$ of a unique $A^{\prime} \in\{\mathbb{R}, \mathbb{C}, \mathbb{H}, \mathbb{O}\}$, i.e. $A=A^{\prime}$ as a vector space, and the multiplication in $A$ is given by

$$
x \cdot y=f(x) g(y)
$$

for all $x, y \in A$, where $f$ and $g$ are linear orthogonal operators on $A$, and juxtaposition is multiplication in $A^{\prime}$.

Moreover, Albert shows that the norm in $A$ coincides with the norm defined in $A^{\prime}$.

Thus the objects of $\mathcal{A}$ are partitioned into four classes according to their dimension, and the class of $d$-dimensional algebras, $d \in\{1,2,4,8\}$, forms a full subcategory $\mathcal{A}_{d}$ of $\mathcal{A}$. For $d>1$ we moreover have the following decomposition due to Darpö and Dieterich [5]. 
Proposition 1.2. Let $A \in \mathcal{A}_{d}$ where $d \in\{2,4,8\}$. For each $a, b \in A \backslash\{0\}$ it holds that $\operatorname{sgn}\left(\operatorname{det}\left(L_{a}\right)\right)=\operatorname{sgn}\left(\operatorname{det}\left(L_{b}\right)\right)$ and $\operatorname{sgn}\left(\operatorname{det}\left(R_{a}\right)\right)=\operatorname{sgn}\left(\operatorname{det}\left(R_{b}\right)\right) .{ }^{1}$ The double sign of $A$ is the pair $(i, j) \in C_{2}^{2}$ where $i=\operatorname{sgn}\left(\operatorname{det}\left(L_{a}\right)\right)$ and $j=\operatorname{sgn}\left(\operatorname{det}\left(R_{a}\right)\right)$ for all $a \in A \backslash\{0\}$. Moreover, for all $d \in\{2,4,8\}$, it holds that

$$
\mathcal{A}_{d}=\coprod_{(i, j) \in C_{2}^{2}} \mathcal{A}_{d}^{i j}
$$

where $\mathcal{A}_{d}^{i j}$ is the full subcategory of $\mathcal{A}_{d}$ formed by all objects having double sign $(i, j)$.

Furthermore, the following has been achieved towards obtaining a complete understanding of the category $\mathcal{A}$.

- A classification of the categories $\mathcal{A}_{1}$ and $\mathcal{A}_{2}$, and a complete description of the set $\mathcal{A}(\mathbb{R}, B)$ for $B \in \mathcal{A}_{2}$.

- A classification of the category $\mathrm{SO}_{3}\left(\mathrm{SO}_{3} \times \mathrm{SO}_{3}\right)$, where the action is by simultaneous conjugation, and a proof that this category is equivalent to $\mathcal{A}_{4}^{k l}$ for any $(k, l) \in C_{2}^{2}$. The equivalence is expressed in terms of a category $\mathcal{C}$ and equivalences $\mathcal{F}^{k l}: \mathcal{C} \rightarrow \mathcal{A}_{4}^{k l}$ and $\mathcal{G}: \mathcal{C} \rightarrow \mathrm{SO}_{3}\left(\mathrm{SO}_{3} \times \mathrm{SO}_{3}\right)$. (See [7].)

- A description of the automorphism groups in $\mathcal{A}_{4}$. (See [7].)

- An explicit description of all those $A \in \mathcal{A}_{4}$ for which there is a morphism $\phi: C \rightarrow A$ for some $C \in \mathcal{A}_{2}$. (See [8].)

- Conditions for when two eight-dimensional algebras are isomorphic [2], and partial classifications of the subcategory $\mathcal{A}_{8}$, see e.g. [4].

In the remainder of this section, the first item in this list will be summarized. Section 2 recollects the results of the second item, and expresses it in terms of a cross-section for $\mathcal{A}_{4}$. The main results of the present article, and consequences thereof, are given in Section 3, where we investigate morphisms from $\mathbb{R}$ to absolute valued algebras of dimension four, and in Section 4, where the same is done for morphisms from two-dimensional absolute valued algebras. In Section 5 we study the irreducibility of the morphisms of Section 3, and in Section 6 we determine of the number of orbits of $\mathcal{A}(C, A)$ for $C \in \mathcal{A}_{2}$ and $A \in \mathcal{A}_{4}$, under the action of the automorphism groups of $C$ and $A$ by composition. The final section supplies technical arguments to carry results that have been obtained for a specific crosssection of $\mathcal{A}_{4}$ to general four-dimensional absolute valued algebras.

1.3. Basic Results. It is known that $\mathcal{A}_{1}$ is classified by $\mathbb{R}$, and that every $C \in \mathcal{A}_{2}$ with double sign $(i, j) \in C_{2}^{2}$ is isomorphic to $\mathbb{C}^{i j}$, this being the algebra with underlying vector space $\mathbb{C}$, and multiplication

$$
(x, y) \mapsto x_{j} y_{i},
$$

where $\forall c \in \mathbb{C}, c_{+}=c$ and $c_{-}=\bar{c}$, and juxtaposition is multiplication in $\mathbb{C}^{2}$

To describe the morphisms from $\mathbb{R}$ to algebras of dimension two, we recall the following result, which will be important in the coming sections.

Proposition 1.3. Let $A$ be a finite dimensional absolute valued algebra, and let $\operatorname{Ip}(A)$ be the set of all idempotents in $A \backslash\{0\}$. Then

\footnotetext{
${ }^{1}$ The sign function $\operatorname{sgn}: \mathbb{R} \backslash\{0\} \rightarrow\{1,-1\}$ is defined by $\operatorname{sgn}(r)=r /|r|$.

${ }^{2}$ The notation $\mathbb{C}^{i j}$ is used due to practical advantages over the standard notation $\mathbb{C}=\mathbb{C}^{++}$, $* \mathbb{C}=\mathbb{C}^{+-}, \mathbb{C}^{*}=\mathbb{C}^{-+}$, and $\stackrel{*}{\mathbb{C}}=\mathbb{C}^{--}$.
} 
(1) $\operatorname{Ip}(A) \neq \emptyset$, and

(2) for each algebra homomorphism $\psi: \mathbb{R} \rightarrow A, \psi(1)$ is an idempotent, and the map $\psi \mapsto \psi(1)$ gives a one-to-one correspondence between $\mathcal{A}(\mathbb{R}, A)$ and $\operatorname{Ip}(A)$.

The first item in fact holds for any finite dimensional non-zero real or complex algebra where $x^{2} \neq 0$ for each $x \neq 0[9]$, and the second is readily checked. For absolute valued algebras of dimension two, it is known that $\operatorname{Ip}\left(\mathbb{C}^{i j}\right)=\{1\}$ for $(i, j) \neq(-,-)$, and $\operatorname{Ip}\left(\mathbb{C}^{--}\right)=\left\{x \in \mathbb{C} \mid x^{3}=1\right\}$. Hence, the category $\mathcal{A}_{\leq d}$ of absolute valued algebras with dimension at most $d$ is understood for $d=2$, and we intend to gain the same understanding of $\mathcal{A}_{\leq 4}$.

\section{Absolute Valued Algebras of Dimension Four}

2.1. Introduction. In view of Proposition 1.2, the category $\mathcal{A}_{4}$ of four-dimensional absolute valued algebras admits the decomposition

$$
\mathcal{A}_{4}=\coprod_{(k, l) \in C_{2}^{2}} \mathcal{A}_{4}^{k l}
$$

where for each $(k, l) \in C_{2}^{2}, \mathcal{A}_{4}^{k l}$ consists of all algebras in $\mathcal{A}_{4}$ with double sign $(k, l)$. Each object in $\mathcal{A}_{4}$ is isomorphic to an object with multiplication defined in terms of quaternion multiplication as follows. [8]

Proposition 2.1. For each $A \in \mathcal{A}_{4}^{k l}$ there exists $A^{\prime}=\left(A^{\prime}, \cdot\right) \in \mathcal{A}_{4}^{k l}$ and $a, b \in \mathbb{S}(\mathbb{H})$, such that $A \simeq A^{\prime}$ and the multiplication - is given by

$$
\begin{array}{ll}
x \cdot y=a x y b & \text { if } \quad(k, l)=(+,+), \\
x \cdot y=\bar{x} a y b & \text { if }(k, l)=(+,-), \\
x \cdot y=a x b \bar{y} & \text { if }(k, l)=(-,+), \text { and } \\
x \cdot y=a \bar{x} \bar{y} b & \text { if } \quad(k, l)=(-,-),
\end{array}
$$

where juxtaposition denotes multiplication in $\mathbb{H}$. Conversely, given any $a, b \in$ $\mathbb{S}(\mathbb{H})$, (2.2) determines the structure of an algebra in $\mathcal{A}_{4}^{k l}$ for each $(k, l) \in C_{2}^{2}$.

An algebra $A^{\prime} \in \mathcal{A}_{4}^{k l}$ with multiplication given by $(2.2)$ for some $a, b \in \mathbb{S}(\mathbb{H})$ will be denoted by $\mathbb{H}^{k l}(a, b)$.

2.2. Classification. It was shown in [7] that for each $(k, l) \in C_{2}^{2}$ there are equivalences of categories

$$
\mathcal{A}_{4}^{k l} \stackrel{\mathcal{F}^{k l}}{\longleftarrow}(\mathbb{S}(\mathbb{H}) \times \mathbb{S}(\mathbb{H})) \stackrel{\mathcal{G}}{\longrightarrow} \mathrm{SO}_{3}\left(\mathrm{SO}_{3} \times S_{3}\right)
$$

where $E=C_{2}^{2} \times(\mathbb{S}(\mathbb{H}) /\{1,-1\})$ acts on $\mathbb{S}(\mathbb{H}) \times \mathbb{S}(\mathbb{H})$ by

$$
E \times(\mathbb{S}(\mathbb{H}) \times \mathbb{S}(\mathbb{H})) \rightarrow \mathbb{S}(\mathbb{H}) \times \mathbb{S}(\mathbb{H}),((\epsilon, \delta, p\{1,-1\}),(a, b)) \mapsto(\epsilon p a \bar{p}, \delta p b \bar{p}),
$$

and $\mathrm{SO}_{3}$ acts on $\mathrm{SO}_{3} \times \mathrm{SO}_{3}$ by simultaneous conjugation

$$
\mathrm{SO}_{3} \times\left(\mathrm{SO}_{3} \times \mathrm{SO}_{3}\right) \rightarrow \mathrm{SO}_{3} \times \mathrm{SO}_{3},(\rho,(\phi, \psi)) \mapsto\left(\rho \phi \rho^{-1}, \rho \psi \rho^{-1}\right) .
$$

The functors $\mathcal{F}^{k l}$ are defined on objects by $\mathcal{F}^{k l}(a, b)=\mathbb{H}^{k l}(a, b)$, and on morphisms by

$$
\mathcal{F}^{k l}(\epsilon, \delta, p\{1,-1\})=\epsilon \delta \kappa_{p} .
$$


The functor $\mathcal{G}$ is defined on objects by $\mathcal{G}(a, b)=\left(\kappa_{a}, \kappa_{b}\right)$, and $\mathcal{G}(\epsilon, \delta, p\{1,-1\})$ is the morphism defined by

$$
(\phi, \psi) \mapsto\left(\kappa_{p} \phi \kappa_{\bar{p}}, \kappa_{p} \psi \kappa_{\bar{p}}\right)
$$

for each $(\phi, \psi) \in S_{3} \times S O_{3}$. The fact that these constructions are well-defined was shown in [7].

We begin by applying the equivalences of categories to express the classification of $\mathrm{SO}_{3}\left(\mathrm{SO}_{3} \times \mathrm{SO}_{3}\right)$, given in [7], as a classification of all four-dimensional absolute valued algebras, i.e. to describe the image of the given cross-section of $\mathrm{SO}_{3}\left(\mathrm{SO}_{3} \times\right.$ $\mathrm{SO}_{3}$ ) under the functor

$$
\mathcal{F}^{k l} \circ \mathcal{H}
$$

for each $(k, l) \in C_{2}^{2}$, where $\mathcal{H}$ is a quasi-inverse functor to $\mathcal{G}$. This is the content of the following result.

Theorem 2.2. Let $u, v \in \mathbb{S}(\Im \mathbb{H})$ be any two orthogonal elements. Let $(k, l) \in C_{2}^{2}$ and $A \in \mathcal{A}_{4}^{k l}$. Then $A \simeq \mathbb{H}^{k l}(a, b)$ where $a, b$ are given by

$$
a=\alpha_{c}+\alpha_{s} u, \quad b=\beta_{c}+\beta_{s}\left(\gamma_{c} u+\gamma_{s} v\right)
$$

for precisely one triple $(\alpha, \beta, \gamma)$ satisfying one of

(1) $(\alpha, \beta, \gamma) \in[0, \pi / 2] \times\{0\} \times\{0\}$,

(2) $(\alpha, \beta, \gamma) \in\{0\} \times(0, \pi / 2] \times\{0\}$,

(3) $(\alpha, \beta, \gamma) \in(0, \pi / 2) \times(0, \pi) \times[0, \pi / 2)$,

(4) $(\alpha, \beta, \gamma) \in\{\pi / 2\} \times(0, \pi / 2] \times[0, \pi / 2)$, or

(5) $(\alpha, \beta, \gamma) \in(0, \pi / 2] \times(0, \pi / 2] \times\{\pi / 2\}$.

Remark 2.3. Note that in case 1 above, the restriction on $\gamma$ is for the sake of uniqueness; indeed, when $\beta=0$, it holds that $b=1$ for any value of $\gamma$. Observe moreover that the five cases are mutually exclusive.

Theorem 2.2 follows from the classification of $\mathrm{SO}_{3}\left(\mathrm{SO}_{3} \times \mathrm{SO}_{3}\right)$ and the explicit description of the equivalences of categories (2.3) given in [7] and quoted above. These use the following fact from [3]: given a quaternion $q=\cos \theta+w \sin \theta$, where $w \in \mathbb{S}(\Im \mathbb{H})$, the map $x \mapsto q x \bar{q}$ is a rotation in $\Im \mathbb{H}$ with axis $w$ and angle of rotation $2 \theta$.

We fix a pair of quaternions $u, v \in \mathbb{S}(\Im \mathbb{H})$ for the sake of definiteness as follows.

Definition 2.4. The set of all $\mathbb{H}^{k l}(a, b) \in \mathcal{A}_{4}$, with $(k, l) \in C_{2}^{2}$ and

$$
a=\alpha_{c}+\alpha_{s} \mathbf{i}, \quad b=\beta_{c}+\beta_{s}\left(\gamma_{c} \mathbf{i}+\gamma_{s} \mathbf{j}\right)
$$

with $(\alpha, \beta, \gamma)$ as in Theorem 2.2, is called the canonical cross-section of $\mathcal{A}_{4}$.

The particular choice of orthogonal quaternions in Definition 2.4 is made in order to simplify calculations, and will be used throughout.

\section{Morphisms from $\mathbb{R}$ to Four-Dimensional Algebras}

3.1. Preparatory Results. We now study morphisms from the unique (up to isomorphism) one-dimensional absolute valued algebra $\mathbb{R}$ to four-dimensional algebras belonging to the canonical cross-section of Definition 2.4, thus acquiring an understanding of $\mathcal{A}(\mathbb{R}, A)$ for each $A \in \mathcal{A}_{4}$. Moreover, the results of Section 
7 below transfer details specific to algebras of the canonical cross-section to any four-dimensional absolute valued algebra given as $\mathbb{H}^{k l}(a, b)$ for some $a, b \in \mathbb{S}(\mathbb{H})$.

By virtue of Proposition 1.3, for each $A \in \mathcal{A}_{4}$, describing $\mathcal{A}(\mathbb{R}, A)$ amounts to describing all non-zero idempotents in $A$. Rewriting the equations (2.2) with $y=x$ we thus see that these idempotents are precisely the non-zero solutions to the quaternion equation

$$
\begin{array}{ll}
x^{2}=\bar{a} x \bar{b} & \text { for } \mathcal{A}_{4}^{++}, \\
x^{2}=a x b & \text { for } \mathcal{A}_{4}^{+-} \text {and } \mathcal{A}_{4}^{-+}, \text {and } \\
\bar{x}^{2}=\bar{a} x \bar{b} & \text { for } \mathcal{A}_{4}^{--} .
\end{array}
$$

To simplify the quadratic terms in the above equations, we recall the notion of a quadratic algebra.

Definition 3.1. An algebra $A$ over a field $k$ is called quadratic if it is non-zero, unital, and if for each $x \in A$ there exist $\lambda, \mu \in k$ such that

$$
x^{2}=\lambda x+\mu 1 \text {. }
$$

Calculating $x^{2}$ for arbitrary $x \in \mathbb{H}$ proves the following result.

Lemma 3.2. $\mathbb{H}$ is quadratic and each $x \in \mathbb{H}$ satisfies $x^{2}=2 \Re(x) x-\|x\|^{2} 1$.

With this in mind, we construct for each real number a set of matrices in $\mathbb{R}^{4 \times 4}$, to be used as the main tool in investigating non-zero idempotents.

Definition 3.3. Given $a, b \in \mathbb{S}(\mathbb{H})$, and $(k, l) \in C_{2}^{2}$, the maps $M_{a, b}^{k l}: \mathbb{R} \rightarrow \mathbb{R}^{4 \times 4}$ are defined by

(1) $M_{a, b}^{++}(r)=2 r I-L_{\bar{a}} R_{\bar{b}}$

(2) $M_{a, b}^{+-}(r)=M_{a, b}^{-+}(r)=2 r I-L_{a} R_{b}$

(3) $M_{a, b}^{--}(r)=2 r K-L_{\bar{a}} R_{\bar{b}}$

for all $r \in \mathbb{R}$, where $I$ is the identity matrix in $\mathbb{R}^{4 \times 4}$ and $K$ the matrix of quaternion conjugation.

Now, due to Lemma 3.2, the following proposition outlines the method that will be used to determine the idempotents. To simplify notation we identify a quaternion $x=r+s_{1} \mathbf{i}+s_{2} \mathbf{j}+s_{3} \mathbf{k}$ with the column matrix $\left(r, s_{1}, s_{2}, s_{3}\right)^{T}$, and use the notation $L_{c}$ and $R_{c}, c \in \mathbb{H}$, also for the matrices in the standard basis of left and right multiplication by $c$, respectively.

Proposition 3.4. Given $(k, l) \in C_{2}^{2}$, and $a, b \in \mathbb{S}(\mathbb{H})$, let $A=\mathbb{H}^{k l}(a, b)$, and let $x=r+s_{1} \mathbf{i}+s_{2} \mathbf{j}+s_{3} \mathbf{k} \in A$. Then

(1) $x \in \operatorname{Ip}(A)$ if and only if $M_{a, b}^{k l}(r) x=1$ and $\|x\|=1$, and

(2) if $A$ belongs to the canonical cross-section, then for each fixed $r$, the quaternion equation $M_{a, b}^{k l}(r) x=1$ is equivalent to a linear system of four real equations in the variables $s_{i}, i \in \underline{3}$.

Proof. We prove the statements for $(k, l)=(+,+)$. The other cases are proven analogously.

(1) We have

$$
M_{a, b}^{++}(r) x=2 r x-\bar{a} x \bar{b} .
$$


Assume that $x \in \mathbb{H}$ satisfies $M_{a, b}^{++}(r) x=1$ and $\|x\|=1$. Then $\bar{a} x \bar{b}=$ $2 r x-1=2 \Re(x) x-\|x\|^{2} 1$, which by virtue of Lemma 3.2 implies the equation (3.1) corresponding to $(k, l)=(+,+)$. Hence $x$ is non-zero and idempotent. Conversely, if $x$ is non-zero and idempotent, then by multiplicativity of the norm, $\|x\|=1$, and

$$
M_{a, b}^{++}(r) x=2 r x-\bar{a} x \bar{b}=2 \Re(x) x-\|x\|^{2} 1+1-\bar{a} x \bar{b}=1+x^{2}-\bar{a} x \bar{b}=1
$$

where the two rightmost equalities follow from Lemma 3.2 and (3.1).

(2) Writing out the equation componentwise, one obtains

$$
\begin{aligned}
2 r^{2}-1 & =\left(\alpha_{c} \beta_{c}-\alpha_{s} \beta_{s} \gamma_{c}\right) r+\left(\alpha_{s} \beta_{c}+\alpha_{c} \beta_{s} \gamma_{c}\right) s_{1} \\
& +\alpha_{c} \beta_{s} \gamma_{s} s_{2}+\alpha_{s} \beta_{s} \gamma_{s} s_{3} \\
2 r s_{1} & =-\left(\alpha_{s} \beta_{c}+\alpha_{c} \beta_{s} \gamma_{c}\right) r+\left(\alpha_{c} \beta_{c}-\alpha_{s} \beta_{s} \gamma_{c}\right) s_{1} \\
& -\alpha_{s} \beta_{s} \gamma_{s} s_{2}+\alpha_{c} \beta_{s} \gamma_{s} s_{3} \\
2 r s_{2} & =-\alpha_{c} \beta_{s} \gamma_{s} r-\alpha_{s} \beta_{s} \gamma_{s} s_{1} \\
& +\left(\alpha_{c} \beta_{c}+\alpha_{s} \beta_{s} \gamma_{c}\right) s_{2}+\left(\alpha_{s} \beta_{c}-\alpha_{c} \beta_{s} \gamma_{c}\right) s_{3} \\
2 r s_{3} & =\alpha_{s} \beta_{s} \gamma_{s} r-\alpha_{c} \beta_{s} \gamma_{s} s_{1} \\
& +\left(\alpha_{c} \beta_{s} \gamma_{c}-\alpha_{s} \beta_{c}\right) s_{2}+\left(\alpha_{c} \beta_{c}+\alpha_{s} \beta_{s} \gamma_{c}\right) s_{3} .
\end{aligned}
$$

Fixing $r$, this is a linear system in $s_{i}, i \in \underline{3}$, with real coefficients.

3.2. Description of Idempotents. In order to describe the idempotents in each four-dimensional absolute valued algebra, we split into cases according to the double sign of the algebra, and determine the non-zero idempotents by solving the equations of Proposition 3.4(1) for the double sign in question. The results are presented below. It turns out that the algebras having double sign $(-,-)$ have substantially different properties with respect to idempotents, and therefore we present this case separately. The computations, however, are analogous to those of the other cases.

3.2.1. Idempotents in $\mathbb{H}^{k l}(a, b)$ with $(k, l) \neq(-,-)$. In this section, the non-zero idempotents are given either explicitly or in terms of roots of a real polynomial. To begin with, this polynomial, together with a number of other functions to be used, are defined.

Definition 3.5. Given $(k, l) \in C_{2}^{2} \backslash\{(-,-)\}$, let $A=\mathbb{H}^{k l}(a, b)$ be in the canonical cross-section with $a, b$ given in terms of $(\alpha, \beta, \gamma)$ by (2.5), and set $\sigma=-k l$. Define $p=p_{a, b}^{k l}, q=q_{a, b}^{k l} \in \mathbb{R}[X]$ and $t_{i}=t_{i, a, b}^{k l} \in \mathbb{R}(X), i \in \underline{3}$, by

$$
\begin{gathered}
p(X)=\left(4 X^{3}-8 \alpha_{c} \beta_{c} X^{2}+\left(4 \alpha_{c}^{2}+4 \beta_{c}^{2}-3\right) X+\alpha_{s} \beta_{s} \gamma_{c}-\alpha_{c} \beta_{c}\right)\left(4 X^{2}-1\right), \\
q(X)=\alpha_{s} \beta_{s} \gamma_{s}\left(8 X^{3}-4\left(3 \alpha_{c} \beta_{c}+\alpha_{s} \beta_{s} \gamma_{c}\right) X^{2}+\left(4 \alpha_{c}^{2}+4 \beta_{c}^{2}-2\right) X\right. \\
\left.+\alpha_{s} \beta_{s} \gamma_{c}-\alpha_{c} \beta_{c}\right), \\
t_{1}(X)=\sigma \alpha_{s} \beta_{s} \gamma_{s} X \frac{\left(\alpha_{s} \beta_{c}+\alpha_{c} \beta_{s} \gamma_{c}\right)\left(4 X^{2}+1\right)-4\left(\alpha_{c} \alpha_{s}+\beta_{c} \beta_{s} \gamma_{c}\right) X}{q(X)} \\
t_{2}(X)=\sigma \alpha_{s} \beta_{s}^{2} \gamma_{s}^{2} X \frac{\alpha_{c}\left(4 X^{2}+1\right)-4 \beta_{c} X}{q(X)}, \quad t_{3}(X)=\alpha_{s}^{2} \beta_{s}^{2} \gamma_{s}^{2} X \frac{4 X^{2}-1}{q(X)} .
\end{gathered}
$$


Using Proposition 3.4(1) to determine the non-zero idempotents, we arrive at the following result.

Theorem 3.6. Given $(k, l) \in C_{2}^{2} \backslash\{(-,-)\}$, let $A=\mathbb{H}^{k l}(a, b)$ be in the canonical cross-section with $a, b$ given in terms of $(\alpha, \beta, \gamma)$ by (2.5), and set $\sigma=-k l$. Let moreover $p, q$ and $t_{i}, i \in \underline{3}$, be given by Definition 3.5 .

(1) If $\gamma=0$, then $x=(\alpha+\beta)_{c}+\sigma(\alpha+\beta)_{s} \mathbf{i}$ is the unique isolated non-zero idempotent in $A$.

(2) If $\gamma=0$ and $\alpha=\beta>\pi / 6$, then the points of the set

$$
\left\{\frac{1}{2}+\sigma \frac{\alpha_{c}}{2 \alpha_{s}} \mathbf{i}+s_{2} \mathbf{j}+s_{3} \mathbf{k} \mid s_{2}^{2}+s_{3}^{2}=1-\frac{1}{4 \alpha_{s}^{2}}\right\}
$$

are precisely the non-isolated idempotents in $A$.

(3) If $\gamma \neq 0$ and $\alpha_{c} \beta_{c}=\alpha_{s} \beta_{s} \gamma_{c}$, then $\sigma \beta_{c} \mathbf{i} / \alpha_{s}+\sigma \alpha_{c} \beta_{s} \gamma_{s} \mathbf{j}-\alpha_{s} \beta_{s} \gamma_{s} \mathbf{k}$ is a non-zero idempotent.

(4) If $\gamma \neq 0$, and $r \in \mathbb{R}$ satisfies

$$
p(r)=0 \neq q(r) \text { and } r^{2}+\sum_{i=1}^{3} t_{i}(r)^{2}=1,
$$

then $r+t_{1}(r) \mathbf{i}+t_{2}(r) \mathbf{j}+t_{3}(r) \mathbf{k}$ is a non-zero idempotent.

(5) Every non-zero idempotent in $A$ is given by precisely one of the cases 1-4.

Proof. We outline the main details of the computations in the case of double sign $(+,+)$, as again the other cases are proven analogously. To this end we solve the equations (3.2)-(3.5) above.

For each fixed $r$, we take three equations among (3.2)-(3.5); our choice will be (3.3)-(3.5). In the variables $s_{i}, i \in \underline{3}$, this gives a system of linear equations with coefficient matrix

$$
M=\left(\begin{array}{ccc}
-\alpha_{s} \beta_{s} \gamma_{s} & \alpha_{c} \beta_{c}+\alpha_{s} \beta_{s} \gamma_{c}-2 r & \alpha_{s} \beta_{c}-\alpha_{c} \beta_{s} \gamma_{c} \\
-\alpha_{c} \beta_{s} \gamma_{s} & \alpha_{c} \beta_{s} \gamma_{c}-\alpha_{s} \beta_{c} & \alpha_{c} \beta_{c}+\alpha_{s} \beta_{s} \gamma_{c}-2 r \\
\alpha_{c} \beta_{c}-\alpha_{s} \beta_{s} \gamma_{c}-2 r & -\alpha_{s} \beta_{s} \gamma_{s} & \alpha_{c} \beta_{s} \gamma_{s}
\end{array}\right)
$$

and right hand side

$$
N=\left(\begin{array}{c}
\alpha_{c} \beta_{s} \gamma_{s} r \\
-\alpha_{s} \beta_{s} \gamma_{s} r \\
\left(\alpha_{s} \beta_{c}+\alpha_{c} \beta_{s} \gamma_{c}\right) r
\end{array}\right)
$$

(Here, the order of the equations has been altered for computational simplicity.) We now aim at solving, for each fixed $r$, the system $M s=N$, with $s=\left(s_{1}, s_{2}, s_{3}\right)^{T}$, using Gauß-Jordan elimination. Thus we must distinguish those cases for which any of the upper left block determinants of $M$ is zero. The block determinants are all non-zero if and only if $0 \notin\{q(r), m(r)\}$, where $m(r)=\alpha_{s} \beta_{s} \gamma_{s}\left(\beta_{c}-2 \alpha_{c} r\right)$, and we thus consider separately the cases

(1) $m(r)=0$,

(2) $m(r) \neq 0, q(r)=0$ and

$$
\begin{aligned}
& \text { i. } n(r)=0 \text {, } \\
& \text { ii. } n(r) \neq 0
\end{aligned}
$$

where $n(r)=\operatorname{det}\left(M_{1} M_{2} N\right)=\alpha_{s} \beta_{s} \gamma_{s} r\left(1-4 r^{2}\right)$, using the notation $M_{i}$ for the $i^{t h}$ column of $M$.

In case 1, Gauß-Jordan elimination cannot be completed straight-forwardly, and in case $2 . \mathrm{i}$, the system $M s=N$ has infinitely many solutions. In both these cases it 
turns out that the equations (3.2)-(3.5), together with the condition $r^{2}+\|s\|^{2}=1$ on the norm, can easily be solved altogether, giving a list $L$ of idempotents for each $(\alpha, \beta, \gamma)$. Computations show that $L$ includes the idempotents of Items 1-3 of Theorem 3.6. In case 2.ii, the system $M s=N$ has no solutions.

If neither case among 1-2.ii holds, then $q(r) \neq 0$ and Gauß-Jordan elimination determines $s_{i}, i \in \underline{3}$ as $s_{i}=t_{i}(r)$, and inserting these into (3.2) gives the equation $p(r)=0$. For each $r$ that solves this equation and satisfies $r^{2}+\|s\|^{2}=1$ it then follows by Proposition 3.4(1) that $r+s_{1} \mathbf{i}+s_{2} \mathbf{j}+s_{3} \mathbf{k}$ is a non-zero idempotent. Moreover, the elements of $L$ that are not given by Items 1-3 are verified to satisfy the conditions of Item 4 . This proves Items 4 and 5 , and the theorem follows.

3.2.2. Idempotents in $\mathbb{H}^{--}(a, b)$. We proceed similarly in the case of the double $\operatorname{sign}(-,-)$.

Definition 3.7. Let $A=\mathbb{H}^{--}(a, b)$ be in the canonical cross-section with $a, b$ given in terms of $(\alpha, \beta, \gamma)$ by (2.5). Define $p^{\prime}=p_{a, b}^{--}, q^{\prime}=q_{a, b}^{--} \in \mathbb{R}[X]$ and $t_{i}^{\prime}=t_{i, a, b}^{--} \in$ $\mathbb{R}(X), i \in \underline{3}$ by

$$
\begin{aligned}
& p^{\prime}(X)=16 X^{5}+16\left(\alpha_{c} \beta_{c}+\alpha_{s} \beta_{s} \gamma_{c}\right) X^{4}-8 X^{3}-8\left(2 \alpha_{c} \beta_{c}+\alpha_{s} \beta_{s} \gamma_{c}\right) X^{2} \\
& +\left(1-4 \alpha_{c}^{2}-4 \beta_{c}^{2}\right) X+\alpha_{s} \beta_{s} \gamma_{c}-\alpha_{c} \beta_{c}, \\
& q^{\prime}(X)=\alpha_{s} \beta_{s} \gamma_{s}\left(8 X^{3}+4\left(3 \alpha_{c} \beta_{c}+\alpha_{s} \beta_{s} \gamma_{c}\right) X^{2}+\left(4 \alpha_{c}^{2}+4 \beta_{c}^{2}-2\right) X\right. \\
& \left.+\alpha_{c} \beta_{c}-\alpha_{s} \beta_{s} \gamma_{c}\right) \\
& t_{1}^{\prime}(X)=\alpha_{s} \beta_{s} \gamma_{s} X \frac{\left(\alpha_{s} \beta_{c}+\alpha_{c} \beta_{s} \gamma_{c}\right)\left(4 X^{2}+1\right)+4\left(\alpha_{c} \alpha_{s}+\beta_{c} \beta_{s} \gamma_{c}\right) X}{q(X)}, \\
& t_{2}^{\prime}(X)=\alpha_{s} \beta_{s}^{2} \gamma_{s}^{2} X \frac{\alpha_{c}\left(4 X^{2}+1\right)+4 \beta_{c} X}{q(X)}, \quad t_{3}^{\prime}(X)=\alpha_{s}^{2} \beta_{s}^{2} \gamma_{s}^{2} X \frac{1-4 X^{2}}{q(X)} .
\end{aligned}
$$

We then use Proposition 3.4(1) to determine the idempotents.

Theorem 3.8. Let $A=\mathbb{H}^{--}(a, b)$ be in the canonical cross-section with $a, b$ given in terms of $(\alpha, \beta, \gamma)$ by (2.5). Let moreover $p^{\prime}, q^{\prime}$ and $t_{i}^{\prime}, i \in \underline{3}$, be given by Definition 3.7.

(1) If $\gamma=0$ and at least one of $\alpha, \beta$ is non-zero, then

$$
x=\cos \left(\frac{2 \pi k+\alpha+\beta}{3}\right)+\sin \left(\frac{2 \pi k+\alpha+\beta}{3}\right) \mathbf{i}
$$

for $k \in \underline{3}$ are precisely the non-zero idempotents in $A$.

(2) If $\alpha=\beta=\gamma=0$, then 1 is the unique isolated non-zero idempotent in $A$, and the points of the set

$$
\left\{-\frac{1}{2}+s_{1} \mathbf{i}+s_{2} \mathbf{j}+s_{3} \mathbf{k} \mid s_{1}^{2}+s_{2}^{2}+s_{3}^{2}=\frac{3}{4}\right\}
$$

are precisely the non-isolated idempotents.

(3) If $\gamma \neq 0$ and $\alpha_{c} \beta_{c}=\alpha_{s} \beta_{s} \gamma_{c}$, then $-\beta_{c} \mathbf{i} / \alpha_{s}-\alpha_{c} \beta_{s} \gamma_{s} \mathbf{j}-\alpha_{s} \beta_{s} \gamma_{s} \mathbf{k}$ is a non-zero idempotent.

(4) If $\gamma \neq 0$ and $\alpha+\beta=\pi$, then

$$
\left\{\frac{1}{2}+\frac{\gamma_{c}+1}{2 \gamma_{s}} e \mathbf{i}+\frac{e}{2} \mathbf{j}+\frac{\gamma_{c}-1}{2 \gamma_{s}} \mathbf{k} \mid e \in \mathbb{R}, e^{2}=\frac{\gamma_{c}-(2 \gamma)_{c}}{\gamma_{c}+1}\right\}
$$


contains precisely two non-zero idempotents.

(5) If $\gamma \neq 0$ and $\alpha=\beta \geq \pi / 6$, then

$$
\left\{-\frac{1}{2}+\frac{\gamma_{c}-1}{2 \gamma_{s}} f \mathbf{i}+\frac{f}{2} \mathbf{j}-\frac{\gamma_{c}+1}{2 \gamma_{s}} \mathbf{k} \mid f \in \mathbb{R}, f^{2}=\frac{\gamma_{c}+(2 \gamma)_{c}}{\gamma_{c}-1}\right\}
$$

contains precisely one non-zero idempotent if $\alpha=\beta=\pi / 6$, and precisely two otherwise.

(6) If $\gamma \neq 0$, and $r \in \mathbb{R}$ satisfies

$$
p^{\prime}(r)=0 \neq q^{\prime}(r) \text { and } r^{2}+\sum_{i=1}^{3} t_{i}^{\prime}(r)^{2}=1,
$$

then $r+t_{1}^{\prime}(r) \mathbf{i}+t_{2}^{\prime}(r) \mathbf{j}+t_{3}^{\prime}(r) \mathbf{k}$ is a non-zero idempotent.

(7) Every idempotent in $A$ is given by precisely one of the cases 1-6.

The proof is analogous to that of Theorem 3.6.

3.3. General Remarks. In this section we comment on the results obtained above, partly in the light of the following result from [2].

Proposition 3.9. The cardinality $|\operatorname{Ip}(A)|$ for an absolute valued algebra $A$ is either odd or infinite. If it is infinite, then $\operatorname{Ip}(A)$ contains a differentiable manifold of positive dimension.

An open question is posed in [2] asking for an upper bound of the number of non-zero idempotents in an arbitrary absolute valued algebra with finitely many idempotents. We are now able to give a precise answer, along with additional information in the cases where the number of idempotents is infinite.

Proposition 3.10. If $A \in \mathcal{A}_{4}$, then $|\operatorname{Ip}(A)| \in\{1,3,5, \infty\}$. All four cases do occur. If $|\operatorname{Ip}(A)|=\infty$, then $\operatorname{Ip}(A)$ contains precisely one isolated element $x$, and an $n$ sphere with all points equidistant from $x$, and with $n=2$ if $(k, l)=(-,-)$, and $n=1$ otherwise.

Proof. Assume first that $A$ belongs to the canonical cross-section of $\mathcal{A}_{4}$. The last statement is a refolmulation of items 1 and 2 of Theorems 3.6 and 3.8, respectively, from which it also follows that the case $|\operatorname{Ip}(A)|=\infty$ does occur. Next we show that $|\operatorname{Ip}(A)|<\infty$ implies $|\operatorname{Ip}(A)| \leq 5$.

Assume hence that $|\operatorname{Ip}(A)|<\infty$. If $A=\mathbb{H}^{k l}(a, b)$ with $a, b$ given in terms of $(\alpha, \beta, \gamma)$ by $(2.5)$, and $\gamma=0$, then it follows from Theorems 3.6 and 3.8 that $A$ has three idempotents if $(k, l)=(-,-)$, and a unique idempotent otherwise. If $\gamma \neq 0$, then the number of idempotents equals the sum of the number of roots of the quintic $p_{a, b}^{k l}$ and the number of idempotents given by Item 3 of Theorem 3.6 (if $(k, l) \neq(-,-)$ ) or Items $3-5$ of Theorem 3.8 (if $(k, l)=(-,-)$ ). However, if $r$ is the real part of $m$ idempotents given by Theorem 3.6(3) or 3.8(3)-(5), then one verifies directly that $(r-X)^{m} \mid p_{a, b}^{k l}(X)$ and that $q_{a, b}^{k l}(r)=0$. Thus $r$ is not the real part of any idempotent given by Theorem 3.6(4) or 3.8(6), and the total number of idempotents does not exceed the number of roots of $p_{a, b}^{k l}$, which is at most five.

Thus by Proposition 3.9, $|\operatorname{Ip}(A)| \in\{1,3,5, \infty\}$ for each $A$ in the canonical crosssection. If $A$ does not belong to the canonical cross-section, then there exists $A^{\prime}$ in the cross-section and an isomorphism $\rho: A^{\prime} \rightarrow A$. The idempotents of $A$ are precisely the images under $\rho$ of the idempotents of $A^{\prime}$, and $|\operatorname{Ip}(A)| \in\{1,3,5, \infty\}$ 
by the above. If moreover $\left|\operatorname{Ip}\left(A^{\prime}\right)\right|=\infty$, then the configuration of the idempotents is preserved under $\rho$, as an isomorphism of absolute valued algebras respects the norm and maps the standard basis to an orthonormal basis in $A$.

Finally, applying Theorem 3.6 to $\mathbb{H}=\mathbb{H}^{++}(1,1)$ and $\mathbb{H}^{++}(\mathbf{i}, \mathbf{j})$, and Theorem 3.8 to $\mathbb{H}^{--}(\mathbf{i}, \mathbf{j})$, one obtains that these algebras have 1,3 and 5 idempotents respectively. This completes the proof.

Remark 3.11. The proposition in fact answers, for the case of dimension four, another open question in [2], namely it gives the number of connected components of $\operatorname{Ip}(A)$ in an absolute valued algebra $A$ with $|\operatorname{Ip}(A)|=\infty$. This number is hence two for all such four-dimensional algebras.

Regarding the quintic polynomials $p_{a, b}^{k l}$, the reader may have noticed that when $(k, l)=(-,-)$, they were not expressed as products of factors of lower degree. This calls for a comment on the issue of their solvability, which we address here.

Proposition 3.12. There exist $a, b \in \mathbb{S}(\mathbb{H})$ such that the polynomial $p_{a, b}^{--}$is not solvable by radicals.

Proof. Construct the polynomial $p_{a, b}^{--}$where

$$
a=\frac{1}{2}+\frac{\sqrt{3}}{2} \mathbf{i}, \quad b=\frac{1}{4}+\frac{\sqrt{15}}{4} \mathbf{j} .
$$

We then have that $P=8 p_{a, b}^{--}$is a polynomial with integer coefficents. We first prove that $P$ is irreducible over $\mathbb{Z}$, by verifying that there exist no $l, m, n \in \mathbb{Z}$, no $Q \in \mathbb{Z}[X]$ of degree 4 and no $R \in \mathbb{Z}[X]$ of degree 3 such that $P(X)=(X+l) Q(X)$ or $P(X)=\left(X^{2}+m X+n\right) R(X) \cdot{ }^{3}$ A well-known result by Gauß implies that $P$ is then irreducible over $\mathbb{Q}$, and hence clearly so is $p_{a, b}^{--}$.

By e.g. determining the zeros of the derivative, it turns out that $p_{a, b}^{--}$has precisely three real roots. By Lemma 14.7 in [10], the Galois group over $\mathbb{Q}$ of an irreducible polynomial of prime degree $p$ with rational coefficients, having precisely two nonreal roots, is the symmetric group on $p$ elements, and the statement follows.

The reader may find the statement of the proposition discouraging. In the search for other methods to solve the idempotency problem, the author has examined available literature on solutions of quadratic equations in $\mathbb{H}$. This examination has indicated that equations of the form $x^{2}+c x d=0$, where $c$ and $d$ are given quaternions (cf. (3.1) above), have been little studied, and an explicit method of finding the solutions seems not to be known. In any case, the above results, even in the cases where Proposition 3.12 holds, are useful to determine whether a given element is an idempotent or not, or to extract various properties of the idempotents.

\section{Morphisms from Two-Dimensional Algebras}

In this section we explicitly determine all morphisms from any of the four nonisomorphic two-dimensional absolute valued algebras $\mathbb{C}^{i j},(i, j) \in C_{2}^{2}$, to any algebra in the canonical cross-section of $\mathcal{A}_{4}$. As in the case of morphisms from $\mathbb{R}$, Section 7 transfers those results of this section which are specific to algebras of the canonical

\footnotetext{
${ }^{3}$ This is done by evaluating both sides of each equation at $X=0$, and those of the second at $X=1$, to obtain a finite list of possible values for $l, m$ and $n$, and then checking that each of these gives a non-zero remainder when $P(X)$ is divided by $X+l$ and $X^{2}+m X+n$, respectively.
} 
cross-section to any four-dimensional absolute valued algebra given as $\mathbb{H}^{k l}(a, b)$ for some $a, b \in \mathbb{S}(\mathbb{H})$.

4.1. Preparatory Results. We start with the following general observation.

Proposition 4.1. Take $\mathbb{C}^{i j} \in \mathcal{A}_{2}$ and let $A=(A, \cdot)$ be a real algebra with $a \in A$. Then there is at most one algebra homomorphism $\phi: \mathbb{C}^{i j} \rightarrow A$ such that $\phi(\mathbf{i})=a$.

Proof. Assume that there are $\phi_{1}$ and $\phi_{2}$ such that $\phi_{1}(\mathbf{i})=\phi_{2}(\mathbf{i})=a$. Then, denoting the multiplication in $\mathbb{C}^{i j}$ by $\circ$, we have, since conjugation is self-inverse, that

$$
\phi_{1}(1)=\phi_{1}(-\mathbf{i i})=-\phi_{1}\left(\mathbf{i}_{j} \circ \mathbf{i}_{i}\right)=-\phi_{1}(\mathbf{i}) \cdot \phi_{1}(\mathbf{i})=-\phi_{2}(\mathbf{i}) \cdot \phi_{2}(\mathbf{i})=\phi_{2}(1)
$$

where juxtaposition is multiplication in $\mathbb{C}$, and for each $c \in \mathbb{C}^{i j}, c_{+}=c$ and $c_{-}=\bar{c}$. Since $\phi_{1}$ and $\phi_{2}$ are linear and the vector space $\mathbb{C}$ is spanned by $\{1, \mathbf{i}\}$, it follows that $\phi_{1}=\phi_{2}$.

Thus the homomorphisms to be treated in this section are determined by the image of the imaginary unit under them. In computations, however, it is often more convenient to use the following characterization of the morphisms.

Proposition 4.2. Let $C=\mathbb{C}^{i j},(i, j) \in C_{2}^{2}$, and let $A=(A, \cdot) \in \mathcal{A}_{4}$. A map $\phi: C \rightarrow A$ is an algebra homomorphism if and only if it is linear and the following conditions hold:

(1) $\phi(1) \cdot \phi(1)=\phi(1)$,

(2) $\phi(1) \cdot \phi(\mathbf{i})=i \phi(\mathbf{i})$,

(3) $\phi(\mathbf{i}) \cdot \phi(1)=j \phi(\mathbf{i})$ and

(4) $\phi(\mathbf{i}) \cdot \phi(\mathbf{i})=-i j \phi(1)$.

Proof. If $\phi$ is a homomorphism, then $\phi$ is linear and respects multiplication. The latter property, together with the definition of the multiplication in $\mathbb{C}^{i j}$, implies the four items above. If $\phi$ is linear, to show that it is a homomorphism we need only show that it respects the multiplication of the elements of a basis of $\mathbb{C}^{i j}$. Choosing the basis $\{1, \mathbf{i}\}$, this is precisely the content of the four items of the proposition.

Since morphisms in $\mathcal{A}$ are always injective, the set $\mathcal{A}\left(\mathbb{C}^{i j}, \mathbb{H}^{k l}(a, b)\right)$ is nonempty if and only if $\mathbb{H}^{k l}(a, b)$ contains a subalgebra isomorphic to $\mathbb{C}^{i j}$. For each $(i, j),(k, l) \in C_{2}^{2},[8]$ gives a list of conditions on $a, b \in \mathbb{S}(\mathbb{H})$ that hold if and only if $\mathbb{H}^{k l}(a, b)$ has a subalgebra $D \simeq \mathbb{C}^{i j}$. We present here its explicit concequences for elements in the canonical cross-section.

Proposition 4.3. Given $(k, l) \in C_{2}^{2}$, let $A=\mathbb{H}^{k l}(a, b)$ be in the canonical crosssection with $a, b$ given in terms of $(\alpha, \beta, \gamma)$ by (2.5). Then there exists a morphism $\phi: \mathbb{C}^{i j} \rightarrow A$ precisely when

$$
\begin{array}{ll}
\text { 1. } \gamma=0, & \text { if }(i, j)=(k, l), \\
\text { 2. } \alpha=\gamma=\pi / 2, & \\
\text { or } \alpha=\pi / 2, \beta=0, & \text { if }(i, j, k, l)=(+,+,+,-) \vee(i, j)=(+,-) \neq(k, l), \\
\text { 3. } \beta=\gamma=\pi / 2 & \\
\text { or } \alpha=0, \beta=\pi / 2, & \text { if }(i, j, k, l)=(+,+,-,+) \vee(i, j)=(-,+) \neq(k, l), \\
\text { 4. } \alpha=\beta=\pi / 2, & \text { if }(i, j, k, l)=(+,+,-,-) \vee(i, j)=(-,-) \neq(k, l) .
\end{array}
$$

The results follow immediately upon applying the conditions in Proposition 3.2 in [8] to the canonical cross-section. 
4.2. Description of Morphisms. Before presenting the complete description of the morphisms, we give the following result, which is meant to give a geometric picture of the set of morphisms from a two-dimensional absolute valued algebra to a four-dimensional.

Theorem 4.4. For any $(i, j),(k, l) \in C_{2}^{2}$ and any $a, b \in \mathbb{S}(\mathbb{H})$, consider $C=\mathbb{C}^{i j}$ and $A=\mathbb{H}^{k l}(a, b)$. Then either the set $\mathcal{A}(C, A)$ is empty, or the map $\mathcal{A}(C, A) \rightarrow$ $A, \phi \mapsto \phi(\mathbf{i})$ induces a bijection

$$
\mathcal{A}(C, A) \rightarrow \bigsqcup_{\mu=1}^{m} \mathbb{S}^{n}
$$

where $m \in\{1,3\}$ is the number of non-zero idempotents in $C$, and $n \in\{0,1,2\}$ satisfies

$$
n= \begin{cases}0 & \text { if } \operatorname{dim}[\Im(a), \Im(b)]=1 \wedge(i, j)=(k, l), \\ 2-\operatorname{dim}[\Im(a), \Im(b)] & \text { otherwise. }\end{cases}
$$

Remark 4.5. The statement that the map $\phi \mapsto \phi(\mathbf{i})$ induces the bijection here means that the image of this map consists of $m$ disjoint $n$-spheres embedded in $A$, and the bijection is obtained by identifying this image with $\bigsqcup_{\mu=1}^{m} \mathbb{S}^{n}$ in a natural way. The theorem follows from the description of the morphisms from each $\mathbb{C}^{i j}$ to each $A=\mathbb{H}^{k l}(a, b)$ in the canonical cross-section, given below, and holds for arbitrary $\mathbb{H}^{k l}(a, b)$ due to the properties of isomorphisms in $\mathcal{A}_{4}$ given in [8] and quoted in Proposition 7.1 below.

Remark 4.6. Section 6 below deals with the orbits of the actions of the automorphism groups of $C$ and $A$ on $\mathcal{A}(C, A)$ by composition. We will briefly return to the above theorem and comment on it in the light of the results obtained there.

We now give the description of the morphisms to algebras in the canonical crosssection, divided into three parts according to the value of $\operatorname{dim}[\Im(a), \Im(b)]$.

Proposition 4.7. Let $C=\mathbb{C}^{i j}$ and let $A=\mathbb{H}^{k l}(a, b)$ be in the canocinal crosssection with $\operatorname{dim}[\Im(a), \Im(b)]=0$. Then

$$
\mathcal{A}(C, A) \neq \emptyset \Longleftrightarrow(i, j)=(k, l) .
$$

In that case $\phi \in \mathcal{A}(C, A)$ if and only if

$$
\phi(\mathbf{i})=\sin \frac{2 \pi \mu}{m}+u \cos \frac{2 \pi \mu}{m}
$$

for some $u \in \mathbb{S}(\Im \mathbb{H})$ and $\mu \in \underline{m}$, where $m=|\operatorname{Ip}(C)|$.

Proposition 4.8. Let $C=\mathbb{C}^{i j}$ and let $A=\mathbb{H}^{k l}(a, b)$ be in the canonical crosssection with $\operatorname{dim}[\Im(a), \Im(b)]=1$ and $(i, j) \neq(k, l)$. If $\mathcal{A}(C, A) \neq \emptyset$, then $\phi \in$ $\mathcal{A}(C, A)$ if and only if

$$
\phi(\mathbf{i})=\sin \frac{2 \pi \mu}{m}+u \cos \frac{2 \pi \mu}{m}
$$

for some $u \in \mathbb{S}(\Im \mathbb{H}) \cap[\Im(a), \Im(b)]^{\perp}$ and $\mu \in \underline{m}$, where $m=|\operatorname{Ip}(C)|$.

Proposition 4.9. Let $C=\mathbb{C}^{i j}$ and let $A=\mathbb{H}^{k l}(a, b)$ be in the canonical crosssection with either $\operatorname{dim}[\Im(a), \Im(b)]=1$ and $(i, j)=(k, l)$, or $\operatorname{dim}[\Im(a), \Im(b)]=0$. If $\mathcal{A}(C, A) \neq \emptyset$, then $\phi \in \mathcal{A}(C, A)$ if and only if

$$
\phi(\mathbf{i})= \pm\left[v \sin \left(\frac{\alpha+\beta-\gamma+2 \pi \mu}{k}\right)+w \cos \left(\frac{\alpha+\beta-\gamma+2 \pi \mu}{m}\right)\right]
$$


for some $\mu \in \underline{m}$, where $m=|\operatorname{Ip}(C)|$, a,b are given in terms of $(\alpha, \beta, \gamma)$ by (2.5), and the pair $(v, w)$ is given by Table 1.

\begin{tabular}{|l|l|l|l|l|}
\hline$(k, l)$ & $(i, j)=(+,+)$ & $(i, j)=(+,-)$ & $(i, j)=(-,+)$ & $(i, j)=(-,-)$ \\
\hline$(+,+)$ & $(1, \mathbf{i})$ & $(\mathbf{i},-\mathbf{k})$ & $(\mathbf{j},-\mathbf{k})$ & $(1,-\mathbf{k})$ \\
$(+,-)$ & $(\mathbf{i}, \mathbf{k})$ & $(1,-\mathbf{i})$ & $(\mathbf{j}, \mathbf{k})$ & $(1,-\mathbf{k})$ \\
$(-,+)$ & $(\mathbf{j}, \mathbf{k})$ & $(\mathbf{i}, \mathbf{k})$ & $(1,-\mathbf{i})$ & $(1,-\mathbf{k})$ \\
$(-,-)$ & $(1, \mathbf{k})$ & $(\mathbf{i},-\mathbf{k})$ & $(\mathbf{j},-\mathbf{k})$ & $(1,-\mathbf{i})$ \\
\hline
\end{tabular}

TABLE 1. The pair $(v, w)$ of Proposition 4.9.

The proofs of Propositions 4.7-4.9 are computationally heavy; we give an outline of the general ideas, and illustrate the computations by an example.

Proof. (Outlined) Take $A \in \mathcal{A}_{4}$ in the canonical cross-section that satisfies any of the conditions of Proposition 4.3. We first determine the idempotents of $A$ by applying Theorem 3.6 or 3.8. It turns out that under the conditions of Proposition 4.3 , the computations are straight-forward as the roots of the polynomials $p_{a b}^{k l}$ of Theorems 3.6 and 3.8 are easily found. Take now $C=\mathbb{C}^{i j}$ for some $(i, j) \in C_{2}^{2}$. According to Item 1 of Proposition 4.2, the set $\{\phi(1) \mid \phi \in \mathcal{A}(C, A)\}$ is a subset of the set of all non-zero idempotents of $A$. Due to Proposition 4.2.(2)-(4), to each non-zero idempotent $y$ we solve the equations

$$
y \cdot x=i x, x \cdot y=j x, x \cdot x=-i j y
$$

for $x$. For each solution $x$ there then exists $\phi \in \mathcal{A}(C, A)$ with $\phi(\mathbf{i})=x$ and $\phi(1)=y$. (If there exist no solutions, then $y$ is not the image of 1 under any morphism in $\mathcal{A}(C, A)$.) Doing this for all idempotents $y \in A$ determines $\mathcal{A}(C, A)$ completely.

As an example we determine $\mathcal{A}\left(\mathbb{C}^{+-}, \mathbb{H}^{-+}(a, b)\right)$ for $\left.\mathbb{H}^{-+}(a, b)\right)$ in the canonical cross-section with $\gamma \neq 0$.

Example 4.10. The cases with $(i, j)=(+,-)$ and $(k, l)=(-,+)$ fall under Item 2 of Proposition 4.3, where we also have $\beta \neq 0$ as $\gamma \neq 0$. Setting thus $\alpha=\gamma=\pi / 2$, we consider Theorem 3.6. The first two items of the theorem give no idempotents, as $\gamma \neq 0$. The third item is applicable, since $\gamma_{c}=\alpha_{c}=0$, and gives the idempotent $\beta_{c} \mathbf{i}-\beta_{s} \mathbf{k}$. In the forth item, we obtain that the roots of $p$ that are not roots of $q$ under the given conditions are $\pm \sqrt{3-4 \beta_{c}^{2}} / 2$ when $\beta \geq \pi / 6$, and none otherwise. Evaluating the functions $t_{i}(r)$ and computing $r^{2}+\sum_{i=1}^{3} t_{i}(r)^{2}$ for each root $r$, we find that there are precisely two additional idempotents

$$
-\beta_{c} \mathbf{j}+\frac{1-2 \beta_{c}^{2}}{2 \beta_{s}} \mathbf{k} \pm \frac{\sqrt{3-4 \beta_{c}^{2}}}{2}\left(1-\frac{\beta_{c}}{\beta_{s}} \mathbf{j}\right)
$$

if $\beta>\pi / 6$, and none otherwise.

Next we solve (4.1) for each idempotent $y$. If $\|x\| \neq 1$, then by multiplicativity of the norm, $x$ does not satisfy the third equation in (4.1). Thus we require $\|x\|=1$, under which condition Lemma 3.2 implies that (4.1) can be rewritten as

$$
a y b=2 \Re(x) x-1, a x b=-x y, a x b=y x .
$$


This is solved by writing each equation componentwise as a system of real equations. For $y=\beta_{c} \mathbf{i}-\beta_{s} \mathbf{k}$, one obtains two solutions $x= \pm\left(\beta_{s} \mathbf{i}+\beta_{c} \mathbf{k}\right)$, while for the other idempotents, no solution exists. Hence for each $\mathbb{H}^{-+}(a, b)$ in the canonical cross-section with $\gamma \neq 0$ we have

$$
\phi \in \mathcal{A}\left(\mathbb{C}^{+-}, \mathbb{H}^{-+}(a, b)\right) \Longleftrightarrow \phi(\mathbf{i}) \in\left\{ \pm\left(\beta_{s} \mathbf{i}+\beta_{c} \mathbf{k}\right)\right\} .
$$

\section{IRREDUCIBILITY}

5.1. Definition and Background. A natural question to ask once a class of morphisms has been described is whether the morphisms are irreducible. To begin with, we quote the definition of irreducibility for division algebras. Recall, to this end, that over any field $k$ the finite dimensional division algebras form a category $\mathcal{D}(k)$, in which the morphisms are the non-zero algebra morphisms. The following definition is due to Dieterich [6].

Definition 5.1. Let $A$ and $B$ be finite dimensional division algebras over a field $k$. A morphism $\psi: A \rightarrow B$ in $\mathcal{D}(k)$ is irreducible if it is not an isomorphism and if for any pair $\left(\psi_{1}, \psi_{2}\right)$ of morphisms in $\mathcal{D}(k)$ such that $\psi=\psi_{2} \psi_{1}$, either $\psi_{1}$ is an isomorphism or $\psi_{2}$ is an isomorphism. $\psi$ is reducible if it is not an isomorphism and not irreducible.

An immediate consequence of the definition, and the injectivity of the morphisms in $\mathcal{D}(k)$, is the following proposition.

Proposition 5.2. Let $A$ and $B$ be finite dimensional division algebras over a field $k$. Then there exists a reducible morphism $\psi: A \rightarrow B$ only if there is a subalgebra $C \subset B$ such that $\operatorname{dim} A<\operatorname{dim} C<\operatorname{dim} B$.

For $A, B \in \mathcal{A}_{\leq 4}$ this implies that all morphisms $A \rightarrow B$ are irreducible in case $\operatorname{dim} A=2$ or $\operatorname{dim} B=2$. It remains to consider the morphisms $\mathbb{R} \rightarrow B$ where $\operatorname{dim} B=4$ and $B$ has a two-dimensional subalgebra. As indicated in the outlined proof of Propositions 4.7-4.9, for such algebras that moreover belong to the canonical cross-section it is straight-forward to determine the idempotents explicitly, and this will be used here to investigate the reducibility of the corresponding morphisms.

5.2. Morphisms from $\mathbb{R}$ to $\mathbb{H}^{k l}(a, b)$ with $(k, l) \neq(-,-)$. Without further ado, we describe the irreducibility of the morphisms from $\mathbb{R}$ to $\mathbb{H}^{k l}(a, b)$. Note that if $\mathbb{H}^{k l}(a, b)$ has a subalgebra isomorphic to $\mathbb{C}^{i j}$ for some $(i, j) \in C_{2}^{2}$, then a morphism from $\mathbb{R}$ to $\mathbb{H}^{k l}(a, b)$ factors over $\mathbb{C}^{i j}$ if and only if it factors over each subalgebra of $\mathbb{H}^{k l}(a, b)$ isomorphic to $\mathbb{C}^{i j}$. In the following, we will use these two equivalent formulations interchangeably.

Proposition 5.3. Given $(k, l) \in C_{2}^{2} \backslash\{(-,-)\}$, let $A=\mathbb{H}^{k l}(a, b)$ with $a, b \in \mathbb{S}(\mathbb{H})$ such that $A$ contains a two-dimensional subalgebra. ${ }^{4}$

(1) If $a$ and $b$ are purely imaginary and orthogonal, then $A$ has a subalgebra isomorphic to $\mathbb{C}^{i j}$ for each $(i, j) \neq(k, l)$, and none isomorphic to $\mathbb{C}^{k l}$, and there are precisely three morphisms $\mathbb{R} \rightarrow A$. All of these are reducible and factor over $\mathbb{C}^{--}$, and precisely one factors over each subalgebra.

\footnotetext{
${ }^{4}$ In other words, assume that $A$ satisfies the conditions of Proposition 3.2 in [8]. If $A$ is in the canonical cross-section, this is equivalent to Proposition 4.3 above.
} 
(2) $\quad i$. If $a$ and $b$ are purely imaginary and proportional, then $A$ has precisely two isomorphism types of two-dimensional subalgebras, and there are uncountably many morphisms $\mathbb{R} \rightarrow A$. All of these are reducible and factor over $\mathbb{C}^{--}$, and only the unique morphism corresponding to the isolated non-zero idempotent in A factors over each subalgebra.

ii. If one of $a$ and $b$ is real and the other purely imaginary, then $A$ has precisely two isomorphism types of two-dimensional subalgebras, and there is precisely one morphism $\mathbb{R} \rightarrow A$. This unique morphism is reducible and factors over each subalgebra.

(3) Otherwise, A has precisely one two-dimensional subalgebra, up to isomorphism. Moreover,

i. if $a$ and $b$ are proportional with $1 / 2<\|\Im(a)\|=\|\Im(b)\|<1$, then there are uncountably many morphisms $\mathbb{R} \rightarrow A$. The unique morphism corresponding to the isolated non-zero idempotent in $A$ is reducible, and all other morphisms are irreducible.

ii. if $a$ and $b$ are orthogonal, one is purely imaginary, and the other has imaginary part $z, 1 / 2<\|z\|<1$, then there are precisely three morphisms $\mathbb{R} \rightarrow A$, and precisely one of these is reducible.

iii. in all other cases, there are precisely three morphisms $\mathbb{R} \rightarrow A$ if both $a$ and $b$ are purely imaginary, and precisely one if not. All of these are reducible.

Proof. A morphism $\psi: \mathbb{R} \rightarrow A$ is reducible if and only if there exists a subalgebra $C \subset A$ of dimension two, and $\phi: C \rightarrow A$, such that $\psi(1)=\phi(z)$ for an idempotent $z \in C$. The result follows for $A$ in the canonical cross-section by checking, for each $\psi: \mathbb{R} \rightarrow A$ and $C=\mathbb{C}^{i j}$, whether or not this condition is satisfied. If $\mathbb{H}^{k l}(c, d)$ is not in the cross-section, then evidently it has the same number of subalgebras and morphisms as its representative, and the morphisms factor in the same way. In addition, the conditions on isomorphisms in $\mathcal{A}_{4}$ quoted in Proposition 7.1 below imply that if $\mathbb{H}^{k l}(c, d) \simeq \mathbb{H}^{k l}(a, b)$, then $\|\Im(c)\|=\|\Im(a)\|$ and $\|\Im(d)\|=\|\Im(b)\|$, and moreover $|\langle c, d\rangle|=|\langle a, b\rangle|$. Hence $\mathbb{H}^{k l}(c, d)$ satisfies the same condition among $1-3$.iii as does $\mathbb{H}^{k l}(a, b)$, and the proof is complete.

Note how the isolated idempotents differ in nature whenever there are infinitely many morphisms, and how the magnitude of the imaginary part is of importance in some cases.

5.3. Morphisms from $\mathbb{R}$ to $\mathbb{H}^{--}(a, b)$. The case of double sign $(-,-)$ exhibits, as the reader may have assumed, several fundamental differences.

Proposition 5.4. Let $A=\mathbb{H}^{--}(a, b)$ with $a, b \in \mathbb{S}(\mathbb{H})$ such that $A$ contains a two-dimensional subalgebra.

(1) If $a$ and $b$ are purely imaginary and orthogonal, then A has a subalgebra isomorphic to $\mathbb{C}^{i j}$ for each $(i, j) \neq(-,-)$, and none isomorphic to $\mathbb{C}^{--}$, and there are precisely five morphisms $\mathbb{R} \rightarrow A$. Of these morphisms precisely one factors over each subalgebra, and all others are irreducible.

(2) If $a$ and $b$ are purely imaginary and proportional, or if one of $a$ and $b$ is real and the other purely imaginary, then A has precisely two isomorphism types of two-dimensional subalgebras, and there are precisely three morphisms $\mathbb{R} \rightarrow A$. All of these are reducible and factor over $\mathbb{C}^{--}$, and precisely one factors over each subalgebra. 
(3) Otherwise, A has precisely one two-dimensional subalgebra, up to isomorphism. Moreover,

$i$. if $a$ and $b$ are real, then there are uncountably many morphisms $\mathbb{R} \rightarrow A$. All of these are reducible.

ii. if $a$ and $b$ are purely imaginary and neither proportional nor orthogonal, then there are precisely five morphisms $\mathbb{R} \rightarrow A$ when $0<|\langle a, b\rangle|<1 / 2$ and precisely three when $1 / 2 \leq|\langle a, b\rangle|<1$. In both cases precisely one of these is reducible.

iii. if $a$ and $b$ are orthogonal, one is purely imaginary, and the other having real part $r$, then there are precisely five morphisms $\mathbb{R} \rightarrow A$ when $0<|r|<1 / 2$ and precisely three when $1 / 2 \leq|r|<1$. In both cases precisely one of these is reducible.

iv. in all other cases, there are precisely three morphisms $\mathbb{R} \rightarrow A$. All of these are reducible.

The proof is analogous to that of Proposition 5.3.

5.4. Morphism Quivers. From Propositions 4.3, 5.3 and 5.4 we extract the following partitioning of the object class of $\mathcal{A}_{4}$.

Corollary 5.5. For each $(k, l) \in C_{2}^{2}$, there exist uncountably many isomorphism classes of objects $A \in \mathcal{A}_{4}^{k l}$ such that each morphism $\psi: \mathbb{R} \rightarrow A$ is irreducible, uncountably many isomorphism classes of objects $A^{\prime} \in \mathcal{A}_{4}^{k l}$ such that there is an irreducible morphism $\psi^{\prime}: \mathbb{R} \rightarrow A^{\prime}$, and a reducible morphism $\psi^{*}: \mathbb{R} \rightarrow A^{\prime}$, and uncountably many isomorphism classes of objects $A^{\prime \prime} \in \mathcal{A}_{4}^{k l}$ such that each morphism $\psi^{\prime \prime}: \mathbb{R} \rightarrow A^{\prime \prime}$ is reducible.

One may further combine Propositions 5.3 and 5.4 with the descriptions of morphisms from one- and two-dimensional to four-dimensional absolute valued algebras, which were given in Sections 3 and 4. In doing so, one obtains a complete picture not only of whether the morphisms from dimension one are reducible or not, but also of the morphisms from dimension two over which the reducible morphisms factor. A way to visualize this is by means of a quiver, the morphism quiver, for each four-dimensional absolute valued algebra $A$. The nodes of the morphism quiver are the non-zero idempotents of all canonical representatives of all subalgebras of $A$, and there exists an arrow from a node $n_{1} \in B_{1}$ to a node $n_{2} \in B_{2}$ if and only if there is an irreducible morphism $\phi: B_{1} \rightarrow B_{2}$ such that $\phi\left(n_{1}\right)=n_{2}$.

Example 5.6. Let $A=\mathbf{H}^{-+}(\mathbf{i}, \mathbf{j})$. Then $A$ satisfies the conditions of Item 1 of Proposition 5.3, and we obtain the following quiver.

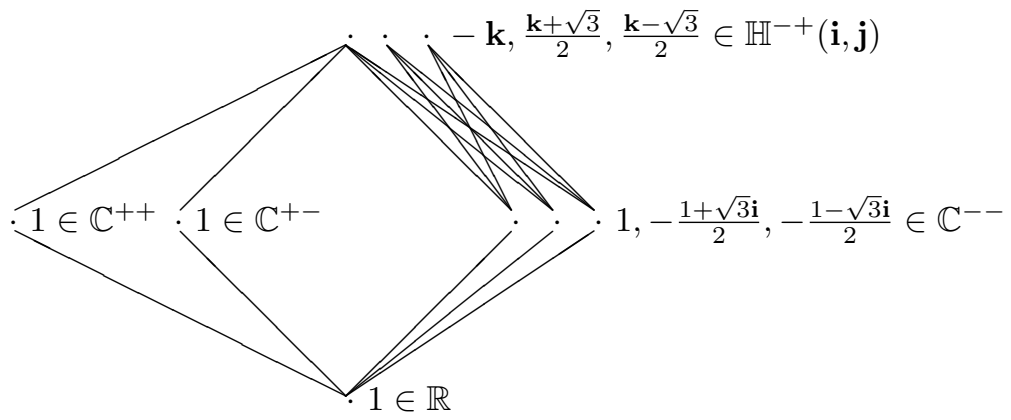


Each arrow is here drawn as a line segment, for visibility, and understood to be directed upwards.

Note that each morphism $\phi: D_{1} \rightarrow D_{2}$, where $D_{1}$ and $D_{2}$ are division algebras over a given field, maps the idempotents of $D_{1}$ injectively to the idempotents of $D_{2}$. The morphism quiver does, as seen from Example 2, not encode which non-zero idempotent $y \in A$ satisfies $y=\phi(x)$ for a given morphism $\phi: \mathbb{C}^{i j} \rightarrow A$ and a given non-zero idempotent $x \in \mathbb{C}^{i j}$, in case there is more than one possibility. Its purpose is to show, for each non-zero idempotent $y \in A$, all possible paths from $1 \in \mathbb{R}$ to $y$, i.e. all possible factorizations of the morphism corresponding to $y$ into irreducible morphisms.

Example 5.7. Let $A=\mathbf{H}^{++}(a, a)$ where $a=\alpha_{c}+\alpha_{s} \mathbf{i}$ and $\pi / 3<\alpha<\pi / 2$, so that $A$ falls under Item 3.1 of Proposition 5.3, and $\operatorname{Ip}(A)$ consists of an isolated point and a circle. The morphism quiver is as follows.

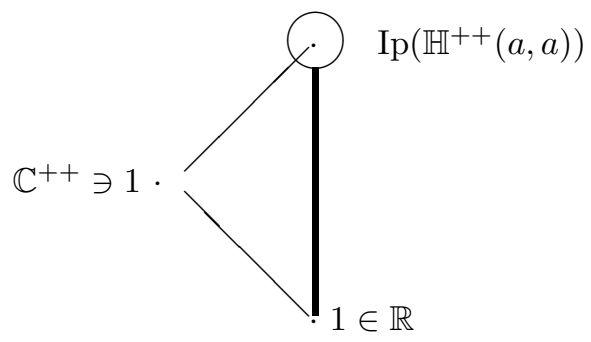

The thickened line segment here means that there is one arrow from $1 \in \mathbb{R}$ to each point on the circle.

Apart from these examples, there are several more different quivers for different $A \in \mathcal{A}_{4}$. The interested reader will have no difficulty to construct these for other algebras in $\mathcal{A}_{4}$.

\section{Action of Automorphism Groups}

The above description of morphisms $\phi \in \mathcal{A}(C, A)$ for $C, A \in \mathcal{A}_{\leq 4}$ was done without regard to the automorphisms of $C$ and $A$. Since for any $\sigma \in \operatorname{Aut}(C)$ and $\tau \in \operatorname{Aut}(A)$ we have $\phi \sigma, \tau \phi \in \mathcal{A}(C, A)$, the automorphism groups of $C$ and $A$ act from the right by precomposition and from the left by postcomposition, respectively. In this section we will consider these two group actions, and determine the number of their orbits. In this context it is natural to also study the left group action

$$
(\operatorname{Aut}(C) \times \operatorname{Aut}(A)) \times \mathcal{A}(C, A) \rightarrow \mathcal{A}(C, A),((\sigma, \tau), \phi) \mapsto \tau \phi \sigma^{-1}
$$

by pre- and postcomposition. The aim of this section is to understand to what extent the properties of the sets $\mathcal{A}(C, A)$ depend on the automorphism groups, and, in a sense, how closely linked the morphisms in $\mathcal{A}(C, A)$ are to each other. We will consider the cases where $C \in \mathcal{A}_{2}$ and $A \in \mathcal{A}_{4}$, as for these cases we have an explicit description of $\mathcal{A}(C, A)$. We start by recalling the structure of the automorphism groups themselves.

6.1. The Automorphism Groups in $\mathcal{A}_{\leq 4}$. For dimensions 1 and 2 , we have the following well-known facts.

Proposition 6.1. Let $C \in \mathcal{A}_{\leq 2}$.

(1) If $C=\mathbb{R}$, then $\operatorname{Aut}(C)$ is trivial. 
(2) If $C=\mathbb{C}^{i j}$ with $(i, j) \neq(-,-)$, then $\operatorname{Aut}(C)$ is generated by complex conjugation.

(3) If $C=\mathbb{C}^{--}$, then $\operatorname{Aut}(C)$ is generated by complex conjugation and rotation by an angle of $2 \pi / 3$.

Thus for $C \in \mathcal{A}_{2}$, Aut $(C)$ has 2 or 6 elements. For dimension 4, the automorphism groups are described for the category $\mathrm{SO}_{3}\left(\mathrm{SO}_{3} \times \mathrm{SO}_{3}\right)$ in [7]. Applying the equivalences of categories in (2.3) to this description gives the following description of the automorphism groups of algebras in the canonical cross-section of $\mathcal{A}_{4}$.

Proposition 6.2. Let $A=\mathbb{H}^{k l}(a, b) \in \mathcal{A}_{4}$ be in the canonical cross-section.

(1) If $\operatorname{dim}[\Im(a), \Im(b)]=0$, then $\operatorname{Aut}(A)=\left\{\kappa_{p} \mid p=\theta_{c}+\theta_{s} q ; \theta \in[0, \pi), q \in\right.$ $\mathbb{S}(\Im \mathbb{H})\}$.

(2) If $\operatorname{dim}[\Im(a), \Im(b)]=1$, let $u \in \mathbb{S}(\Im \mathbb{H})$ be a basis vector of $[\Im(a), \Im(b)]$. Then if

i. at least one of $a, b$ is neither real nor purely imaginary, then $\operatorname{Aut}(A)=\left\{\kappa_{p} \mid p=\theta_{c}+\theta_{s} u ; \theta \in[0, \pi)\right\}$.

ii. each of $a, b$ is either real or purely imaginary, then $\operatorname{Aut}(A)=\left\{\kappa_{p} \mid\right.$ $\left.p=\theta_{c}+\theta_{s} u ; \theta \in[0, \pi)\right\} \cup\left\{\epsilon \kappa_{q} \mid q \in \mathbb{S}(\Im \mathbb{H}) \cap u^{\perp}\right\}$, where $\epsilon=1$ if both a and $b$ are purely imaginary, and -1 otherwise.

(3) If $\operatorname{dim}[\Im(a), \Im(b)]=2$ and

$i$. either non of $a, b$ is purely imaginary, or precisely one of $a, b$ is purely imaginary and $\Im(a), \Im(b)$ are not orthogonal, then $\operatorname{Aut}(A)$ is trivial.

ii. precisely one of $a, b$ is purely imaginary and $\Im(a), \Im(b)$ are orthogonal, then $\operatorname{Aut}(A)=\left\{\mathrm{Id},-\kappa_{v}\right\}$ where $v \in \mathbb{S}(\Im \mathbb{H})$ is a basis vector of the imaginary part of the non-purely imaginary element in $\{a, b\}$.

iii. a, $b$ are both purely imaginary and not orthogonal, then $\operatorname{Aut}(A)=$ $\left\{\mathrm{Id}, \kappa_{w}\right\}$, where $w \in \mathbb{S}(\Im \mathbb{H})$ is orthogonal to $a$ and $b$.

iv. $a, b$ are both purely imaginary and orthogonal, then $\operatorname{Aut}(A)=$ $\left\{\mathrm{Id},-\kappa_{a},-\kappa_{b}, \kappa_{w}\right\}$, where $w \in \mathbb{S}(\Im \mathbb{H})$ is orthogonal to $a$ and $b$.

Remark 6.3. If $A=\mathbb{H}^{k l}(c, d) \simeq \mathbb{H}^{k l}(a, b)=A^{\prime}$, where $A^{\prime}$ is in the canonical cross-section and $A$ is not, then due to the properties of isomorphisms quoted in Proposition 7.1 below, $A$ satisfies the conditions for the same item among 1-3.iv as does $A^{\prime}$. Obviously $\operatorname{Aut}(A) \simeq \operatorname{Aut}\left(A^{\prime}\right)$, but the explicit description of $\operatorname{Aut}(A)$ may differ from that given above for $\operatorname{Aut}\left(A^{\prime}\right)$.

6.2. Orbits of the Actions. We now use the results of Section 6.1 to determine the number of orbits of the three group actions given above on the set $\mathcal{A}(C, A)$ for all $C \in \mathcal{A}_{2}$ and $A \in \mathcal{A}_{4}$. We thus denote by $n_{C}$ the number of orbits of the right action of $\operatorname{Aut}(C)$ by precomposition, by $n_{A}$ the number of orbits of the left action of $\operatorname{Aut}(A)$ by postcomposition, and by $n_{C A}$ the number of orbits of the left action of $\operatorname{Aut}(C) \times \operatorname{Aut}(A)$ by pre- and postcomposition.

Proposition 6.4. Let $C \in \mathcal{A}_{2}$ and $A \in \mathcal{A}_{4}$. Then $n_{C A}=1$ and the pair $\left(n_{C}, n_{A}\right)$ attains one of

$$
(1,1),(1,2),(1,3),(1,6),(\infty, 1),(\infty, 3)
$$

All of these pairs do occur for suitable $C \in \mathcal{A}_{2}$ and $A \in \mathcal{A}_{4}$.

Proof. Using Propositions 6.1 and 6.2, for each $C=\mathbb{C}^{i j}$ and each $A$ in the canonical cross-section, the set $\mathcal{A}(C, A)$ can be partitioned into the equivalence classes of one of the following equivalence relations 
(1) $\phi \sim_{1} \psi \Leftrightarrow \exists \sigma \in \operatorname{Aut}(C) ; \psi=\phi \sigma$,

(2) $\phi \sim_{2} \psi \Leftrightarrow \exists \tau \in \operatorname{Aut}(A) ; \psi=\tau \phi$.

Computing the number of equivalence classes of each relation gives the pair $\left(n_{C}, n_{A}\right)$.

If either $n_{C}=1$ or $n_{A}=1$, then $n_{C A}=1$. If not, then by the previous step, $\left(n_{C}, n_{A}\right)=(\infty, 3)$. Denoting the three $\operatorname{Aut}(A)$-orbits by $\omega_{i}, i \in \underline{3}$, taking an arbitrary $\phi \in \omega_{1}$, and precomposing $\phi$ by each of the (at most) six elements in $\operatorname{Aut}(C)$, one finds that there exist $\rho, \sigma \in \operatorname{Aut}(C)$ such that $\phi \rho \in \omega_{2}$ and $\phi \sigma \in \omega_{3}$. Hence there is one single orbit.

For algebras not in the canonical cross-section, the result holds by applying the above to their canonical representatives, as the number of orbits of any of the three actions involved is preserved under isomorphism.

The computations of the proof of Proposition 6.4, together with Remark 6.3, in fact prove the following, more precise statements.

Proposition 6.5. Let $C \in \mathcal{A}_{2}$ and $A \in \mathcal{A}_{4}$.

(1) The number $n_{C}$ of orbits of the right action of $\operatorname{Aut}(C)$ by precomposition is 1 if $\mathcal{A}(C, A)$ is finite, and $\infty$ otherwise.

(2) The number $n_{A}$ of orbits of the left action of $\operatorname{Aut}(A)$ by postcomposition equals $|\operatorname{Ip}(C)|$ if $A$ 's representative in the canonical cross-section satisfies 1, 2.ii or 3.iv of Proposition 6.2, and $2|\operatorname{Ip}(C)|$ if it satisfies 2.i, 3.ii or 3.iii. In particular, if $\mathcal{A}(C, A)$ is infinite, then $n_{A}=|\operatorname{Ip}(C)|$.

Case 3.i does not occur for those four-dimensional algebras that have twodimensional subalgebras.

Proposition 6.5 partly explains the geometric situation presented in Theorem 4.4. Namely, when $n \in\{1,2\}$, each $n$-sphere corresponds to an orbit of the $\operatorname{Aut}(A)$ action, while each orbit of the $\operatorname{Aut}(C)$-action consists of a pair of points on each $n$-sphere. For the $\operatorname{Aut}(C)$-action the same holds in the case $n=0$, whence all morphisms belong to the same orbit of this action.

\section{Isomorphisms to the CANonical Cross-Section}

Some results above were only formulated for algebras in the canonical crosssection. In order to extend these to more general objects, either the descriptions have to be generalized, or, given $A \in \mathcal{A}_{4}$, one has to explicitly construct an isomorphism to the algebra in the canonical cross-section isomorphic to $A$.

The first approach involves computational difficulties, as the computations of the morphisms, conducted in Sections 3 and 4 above, have depended strongly on the simplifications associated with the particular choice of a cross-section. We therefore devote this section to the second approach; hence, given an algebra $A=$ $\mathbb{H}^{k l}(c, d) \in \mathcal{A}_{4}$ with arbitrary $c, d \in \mathbb{S}(\mathbb{H})$, we determine its representative in the canonical cross-section, and construct an isomorphism. We begin by the following result from [8], supplemented in [7].

Proposition 7.1. Two four-dimensional absolute valued algebras $\mathbb{H}^{k l}(a, b)$ and $\mathbb{H}^{k^{\prime} l^{\prime}}(c, d)$, with $a, b, c, d \in \mathbb{S}(\mathbb{H})$, are isomorphic if and only if $\left(k^{\prime}, l^{\prime}\right)=(k, l)$ and there exists $p \in \mathbb{S}(\mathbb{H})$ and $(\epsilon, \delta) \in C_{2}^{2}$ such that $c=\epsilon p a \bar{p}$ and $d=\delta p b \bar{p}$. In that case, every isomorphism $\psi: \mathbb{H}^{k l}(a, b) \rightarrow \mathbb{H}^{k l}(c, d)$ is of the form $x \mapsto \epsilon \delta p x \bar{p}$. 
Note that Proposition 7.1 is not constructive, as $p$ is not given explicitly. We begin our explicit construction by determining the representatives in the crosssection.

Lemma 7.2. Let $A=\mathbb{H}^{k l}(c, d)$ with $c, d \in \mathbb{S}(\mathbb{H})$ be given. Then the representative of $A$ in the canonical cross-section is $\mathbb{H}^{k l}(a, b)$, with $a, b$ given in terms of $(\alpha, \beta, \gamma)$ by (2.5), where

(1) $\alpha$ is determined uniquely by $\alpha_{c}=|\Re(c)|$,

(2) $\beta$ is determined uniquely by

$$
\beta_{c}= \begin{cases}\operatorname{sgn}(\Re(c)) \operatorname{sgn}(\langle\Im(c), \Im(d)\rangle) \Re(d) & \text { if } 0 \notin\{\Re(c),\langle\Im(c), \Im(d)\rangle\}, \\ |\Re(d)| & \text { otherwise, and }\end{cases}
$$

(3) $\gamma$ is then determined uniquely by $\alpha_{s} \beta_{s} \gamma_{c}=|\langle\Im(c), \Im(d)\rangle|$ if $0 \notin\{\alpha, \beta\}$, and $\gamma=0$ otherwise.

Proof. By Proposition 7.1, there exists $p \in \mathbb{S}(\mathbb{H})$ such that $c=\epsilon p a \bar{p}$ and $d=\delta p b \bar{p}$ for some $(\epsilon, \delta) \in C_{2}^{2}$. Since conjugation by $p$ preserves the real part of a quaternion, we have $\alpha_{c}=\epsilon \Re(c)$ and $\beta_{c}=\delta \Re(d)$, hence $\left|\alpha_{c}\right|=|\Re(c)|$ and $\left|\beta_{c}\right|=|\Re(d)|$. By Theorem 2.2, $0 \leq \alpha \leq \pi / 2$, whence $\alpha_{c}$ is non-negative and determines $\alpha$, which proves 1.

As for 2 , the inner product on $\mathbb{H}$ is preserved under conjugation by a unit norm quaternion, and hence $\langle\Im(c), \Im(d)\rangle=\epsilon \delta \alpha_{s} \beta_{s} \gamma_{c}$. Suppose that $\Re(c) \neq 0$ and $\langle\Im(c), \Im(d)\rangle \neq 0$. Then $\epsilon=\operatorname{sgn}(\Re(c))$ by the above, and $\alpha_{s} \beta_{s} \gamma_{c} \neq 0$, hence positive by Theorem 2.2. Now $\langle\Im(c), \Im(d)\rangle=\epsilon \delta \alpha_{s} \beta_{s} \gamma_{c}$ impies that $\delta=$ $\operatorname{sgn}(\Re(c)) \operatorname{sgn}(\langle\Im(c), \Im(d)\rangle)$, and by the above $\beta_{c}=\delta \Re(d)$.

If $\Re(c)=0$ or $\langle\Im(c), \Im(d)\rangle=0$, i.e. if $\alpha_{c}=0$ or $\alpha_{s} \beta_{s} \gamma_{c}=0$, then Theorem 2.2 implies that $0 \leq \beta \leq \pi / 2$, and thus $\beta_{c}$ is non-negative. Since in all cases $0 \leq \beta \leq \pi$, $\beta_{c}$ determines $\beta$ completely.

Regarding 3, if any of $\alpha$ or $\beta$ vanishes, then so does $\gamma$ by Theorem 2.2. If both $\alpha$ and $\beta$ are non-zero, then $\langle\Im(c), \Im(d)\rangle=\epsilon \delta \alpha_{s} \beta_{s} \gamma_{c}$ determines $\gamma_{c}$ up to sign. Finally, $0 \leq \gamma \leq \pi / 2$ implies that $\gamma_{c}$ is non-negative and determines $\gamma$ completely.

The construction of the isomorphisms relies on the following detail.

Lemma 7.3. Assume that two quaternions $x=s_{1} \mathbf{i}+s_{2} \mathbf{j}+s_{3} \mathbf{k}$ and $y=t \mathbf{i}, t>0$ satisfy $\|x\|=\|y\| \leq 1$. Then

(1) if $s_{2}=s_{3}=0$, then $\left|s_{1}\right|=|t|$; if $s_{1}=t$, then $p=1$ satisfies $x=p y \bar{p}$, and if $s_{1}=-t$, then $p=\mathbf{j}$ satisfies $x=p y \bar{p}$;

(2) otherwise

$$
p=\sqrt{\frac{t+s_{1}}{2 t}}-s_{3} \sqrt{\frac{t-s_{1}}{2 t\left(s_{2}^{2}+s_{3}^{2}\right)}} \mathbf{j}+s_{2} \sqrt{\frac{t-s_{1}}{2 t\left(s_{2}^{2}+s_{3}^{2}\right)}} \mathbf{k}
$$

satisfies $x=p y \bar{p}$.

Note that in $2, p$ is well-defined as $t \pm s_{1} \geq 0$ follows from $\|x\|=\|y\|$, and $s_{2}^{2}+s_{3}^{2} \neq 0$.

Proof. Since $\|x\|=\|y\|$, there exists a rotation in $\Im \mathbb{H}$ that takes $y$ to $x$. Computing the angle and axis of the rotation by elementary linear algebra, the result follows from the fact that if $u \in \mathbb{S}(\Im \mathbb{H})$, then $q=\theta_{c}+\theta_{s} u$ satisfies that $z \mapsto q z \bar{q}$ is a rotation with angle $2 \theta$ around $u$. (The claim can also be verified by direct computation.) 
Once the representative of $A=\mathbb{H}^{k l}(c, d)$ in the canonical cross-section has been determined by Lemma 7.2, the following proposition gives an explicit construction of an isomorphism to $A$ from its representative.

Proposition 7.4. Let $A=\mathbb{H}^{k l}(c, d)$ with $c, d \in \mathbb{S}(\mathbb{H})$ and let $\mathbb{H}^{k l}(a, b)$ be the representative of $A$ in the canonical cross-section, with a, $b$ given in terms of $(\alpha, \beta, \gamma)$ by (2.5).

If $0 \in\{\alpha, \beta, \gamma\}$, then the map $\rho: \mathbb{H}^{k l}(a, b) \rightarrow A, z \mapsto \epsilon \delta p z \bar{p}$ is an isomorphism, where $(\epsilon, \delta) \in C_{2}^{2}$ and $p \in \mathbb{S}(\mathbb{H})$ are given as follows.

(1) If $\alpha=\beta=0$, then $\epsilon=\operatorname{sgn}(\Re(c)), \delta=\operatorname{sgn}(\Re(d))$, and $p=1$.

(2) If $\alpha=0$ and $\beta \neq 0$, then $\epsilon=\operatorname{sgn}(\Re(c))$, and

i. if $\beta \neq \pi / 2$, then $\delta=\operatorname{sgn}(\Re(d))$,

ii. if $\beta=\pi / 2$, then $\delta$ can be chosen freely,

and $p$ is given by Lemma 7.3 upon setting $y=\Im(b)$ and $x=\delta \Im(d)$.

(3) If $\alpha \neq 0$ and $\beta=0$, then $\delta=\operatorname{sgn}(\Re(d))$, and

i. if $\alpha \neq \pi / 2$, then $\epsilon=\operatorname{sgn}(\Re(c))$,

ii. if $\alpha=\pi / 2$, then $\epsilon$ can be chosen freely,

and $p$ is given by Lemma 7.3 upon setting $y=\Im(a)$ and $x=\epsilon \Im(c)$.

(4) If $0 \notin\{\alpha, \beta\}$ and $\gamma=0$, then

i. if $\alpha \neq \pi / 2$, then $\epsilon=\operatorname{sgn}(\Re(c))$ and $\delta=\epsilon \operatorname{sgn}(\langle\Im(c), \Im(d)\rangle)$,

ii. if $\alpha=\pi / 2$ and $\beta \neq \pi / 2$, then $\delta=\operatorname{sgn}(\Re(d))$ and $\epsilon=\delta \operatorname{sgn}(\langle\Im(c), \Im(d)\rangle)$,

iii. if $\alpha=\beta=\pi / 2$, then $\epsilon$ can be chosen freely, and $\delta=\epsilon \operatorname{sgn}(\langle\Im(c), \Im(d)\rangle)$,

and $p$ is given by Lemma 7.3 upon setting $y=\Im(a)$ and $x=\epsilon \Im(c)$.

If $0 \notin\{\alpha, \beta, \gamma\}$, then $\rho: \mathbb{H}^{k l}(a, b) \rightarrow A$, defined by

$$
\mathbf{i} \mapsto \frac{\delta \Im(c)}{\alpha_{s}}, \quad \mathbf{j} \mapsto \frac{\epsilon \alpha_{s} \Im(d)-\delta \beta_{s} \gamma_{c} \Im(c)}{\alpha_{s} \beta_{s} \gamma_{s}}
$$

is an isomorphism, where

(1) if $\alpha \neq \pi / 2$, then $\epsilon=\operatorname{sgn}(\Re(c))$ and

i. if $\beta \neq \pi / 2$, then $\delta=\operatorname{sgn}(\Re(d)) \operatorname{sgn}\left(\beta_{c}\right)$,

ii. if $\beta=\pi / 2$ and $\gamma \neq \pi / 2$, then $\delta=\epsilon \operatorname{sgn}(\langle\Im(c), \Im(d)\rangle)$,

iii. if $\beta=\gamma=\pi / 2$, then $\delta$ can be chosen freely;

(2) if $\alpha=\pi / 2 \notin\{\beta, \gamma\}$, then $\delta=\operatorname{sgn}(\Re(d))$ and $\epsilon=\delta \operatorname{sgn}(\langle\Im(c), \Im(d)\rangle)$;

(3) if $\alpha=\pi / 2$ and $\pi / 2 \in\{\beta, \gamma\}$, then $\epsilon$ can be chosen freely, and

i. if $\beta=\pi / 2 \neq \gamma$, then $\delta=\epsilon \operatorname{sgn}(\langle\Im(c), \Im(d)\rangle)$,

ii. if $\gamma=\pi / 2 \neq \beta$, then $\delta=\operatorname{sgn}(\Re(d))$,

iii. if $\beta=\gamma=\pi / 2$, then $\delta$ can be chosen freely.

Remark 7.5. The fact that conjugation by a unit norm quaternion preserves the real part and inner product implies that $\Re(c), \Re(d)$ and $\langle\Im(c), \Im(d)\rangle$ are non-zero whenever this is required for the sign function to be defined, and that Lemma 7.3 is applicable wherever claimed.

Proof. To prove the statements where $0 \in\{\alpha, \beta, \gamma\}$ it suffices, by Proposition 7.1, to check that the given $\epsilon, \delta$ and $p$ satisfy $c=\epsilon p a \bar{p}$ and $d=\delta p b \bar{p}$, which is straightforward. 
For the cases where $0 \notin\{\alpha, \beta, \gamma\}$, we instead use that by Proposition 7.1 there exist such $\epsilon, \delta$ and $p$, and that an isomorphism is given by $z \mapsto \epsilon \delta p z \bar{p}$. The image of $\mathbf{i}$ under this isomorphism is then determined by $\Im(c)=\epsilon p \Im(a) \bar{p}$, since $\Im(a)=\alpha_{s} \mathbf{i}$ with $\alpha_{s} \neq 0$. This, together with $\Im(d)=\epsilon p \Im(b) \bar{p}$, determines the image of $\mathbf{j}$ since $\Im(b)=\beta_{s} \gamma_{c} \mathbf{i}+\beta_{s} \gamma_{s} \mathbf{j}$ with $\beta_{s} \gamma_{s} \neq 0$. The listed values of $\epsilon$ and $\delta$ are readily checked. Since by Theorem 2.2 there are no more cases, the proof is complete.

Note how the construction of an isomorphism involves a number of choices, and different choices may give different isomorphisms. This is of no importance in this context, as any morphism $\phi$ from an absolute valued algebra $C$ to an algebra $A=\mathbb{H}^{k l}(c, d) \simeq \mathbb{H}^{k l}(a, b)=A^{\prime}$ factors uniquely over any isomorphism $\rho: A^{\prime} \rightarrow A$.

Acknowledgements. The author would like to thank Professor Ernst Dieterich at Uppsala University for valuable discussions and remarks.

\section{REFERENCES}

[1] A. A. Albert, Absolute Valued Real Algebras, Ann. of Math. 48 (1947), pp. 495-501.

[2] A. Calderón, A. Kaidi, C. Martín, A. Morales, M. Ramírez \& A. Rochdi, Finite-Dimensional Absolute Valued Algebras, to appear in Israel J. Math.

[3] J. H. Conway \& D. A. Smith, On Quaternions and Octonions: Their Geometry, Arithmetic, and Symmetry, A K Peters Ltd., Natick (MA), the USA.

[4] J. A. Cuenca Mira, E. Darpö \& E. Dieterich, Classification of the Finite Dimensional Absolute Valued Algebras having a Non-Zero Central Idempotent or a One-Sided Unity, Bull. Sci. Math. 134 (2010), pp. 247-277.

[5] E. Darpö \& E. Dieterich, The Double Sign of a Real Division Algebra of Finite Dimension Greater than One, in preparation.

[6] E. Dieterich, A General Approach to Finite Dimensional Division Algebras, in preparation.

[7] L. Forsberg, Four-Dimensional Absolute Valued Algebras, U.U.D.M. Project Report 2009:9, Department of Mathematics, Uppsala University, Uppsala, Sweden. Link: http://urn.kb.se/resolve?urn=urn:nbn:se:uu:diva-119808

[8] M. I. Ramírez, On Four-Dimensional Absolute Valued Algebras, Proceedings of the International Conference on Jordan Structures, Univ. Málaga, Málaga, Spain, 1999, pp.frm[o]-69173.

[9] B. Segre, La teoria delle algebre ed alcune questioni di realità (Italian), Univ. Roma. Ist. Naz. Alta Mat. Rend. Mat. e Appl. 13 (1954), pp. 157-188.

[10] I. Stewart, Galois Theory, Chapman \& Hall, the USA, (1989).

Uppsala University, Department of Mathematics, P.O. Box 480, SE-75106 Uppsala, SWEDEN 